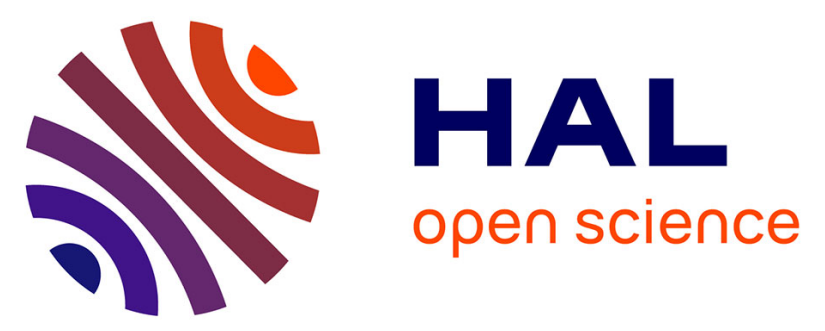

\title{
An annotated checklist of the jumping plant-lice (Insecta: Hemiptera: Psylloidea) from the Mercantour National Park, with seven new records for France and one new synonymy
}

\author{
David Ouvrard, Daniel Burckhardt, Christian Cocquempot
}

\section{To cite this version:}

David Ouvrard, Daniel Burckhardt, Christian Cocquempot. An annotated checklist of the jumping plant-lice (Insecta: Hemiptera: Psylloidea) from the Mercantour National Park, with seven new records for France and one new synonymy. Zoosystema, 2015, 37 (1), pp.251 - 271. 10.5252/z2015n1a13 . hal-01218257

\section{HAL Id: hal-01218257 \\ https://hal.science/hal-01218257}

Submitted on 20 Oct 2015

HAL is a multi-disciplinary open access archive for the deposit and dissemination of scientific research documents, whether they are published or not. The documents may come from teaching and research institutions in France or abroad, or from public or private research centers.
L'archive ouverte pluridisciplinaire HAL, est destinée au dépôt et à la diffusion de documents scientifiques de niveau recherche, publiés ou non, émanant des établissements d'enseignement et de recherche français ou étrangers, des laboratoires publics ou privés. 


\title{
An annotated checklist of the jumping plant-lice (Insecta: Hemiptera: Psylloidea) from the Mercantour National Park, with seven new records for France and one new synonymy
}

\author{
David OUVRARD \\ Department of Life Sciences, Natural History Museum, \\ Cromwell Road, London SW7 5BD (United Kingdom) \\ d.ouvrard@nhm.ac.uk \\ Daniel BURCKHARDT \\ Naturhistorisches Museum, Augustinergasse 2, \\ $\mathrm{CH}-4001$ Basel (Switzerland) \\ daniel.burckhardt@bs.ch \\ Christian COCQUEMPOT \\ CBGP - INRA, 755 avenue d'Agropolis, \\ Campus International de Baillarguet, CS 30016 , \\ 34988 Montférrier-sur-Lez (France) \\ cocquemp@supagro.inra.fr
}

Published on 27 March 2015

KEY WORDS

Psyllids,

Sternorrhyncha,

faunistics,

host plants,

France,
Alps,

new records.

Ouvrard D., Burckhardt D. \& Cocquempot C. 2015. - An annotated checklist of the jumping plant-lice (Insecta: Hemiptera: Psylloidea) from the Mercantour National Park, with seven new records for France and one new synonymy, in Daugeron C., Deharveng L., Isaia M., Villemant C. \& Judson M. (eds), Mercantour/Alpi Marittime All Taxa Biodiversity Inventory. Zoosystema 37 (1): 251-271. http://dx.doi.org/10.5252/z2015n1a13

\section{ABSTRACT}

A total of 68 psyllid species are listed from the Mercantour National Park in Southeast France, where a targeted collecting campaign was conducted between 2009 and 2012, as part of the project "ATBI+M" Mercantour. The insects were collected using Malaise traps, flight intercept traps and sweep nets to sample in the vegetation. Additional information on distribution, biology and host-plants is provided for each species. Seven species are recorded for the first time from France: Craspedolepta artemisiae (Foerster, 1848), Craspedolepta nebulosa (Zetterstedt, 1828), Cacopsylla propinqua (Schaefer, 1949), Cyamophila prohaskai (Priesner, 1927), Eryngiofaga cf. refuga (Loginova, 1966), Bactericera parastriola Conci, Ossiannilsson \& Tamanini, 1988 and Trioza flixiana Burckhardt \& Lauterer, 2002. Trioza (Trioza) rapisardai Conci \& Tamanini, 1984 is a new subjective synonym of Trioza brachyceraea Hodkinson \& White, 1979, which was previously known only from the male holotype. The abundance, distribution and introduction status of some species are discussed. 


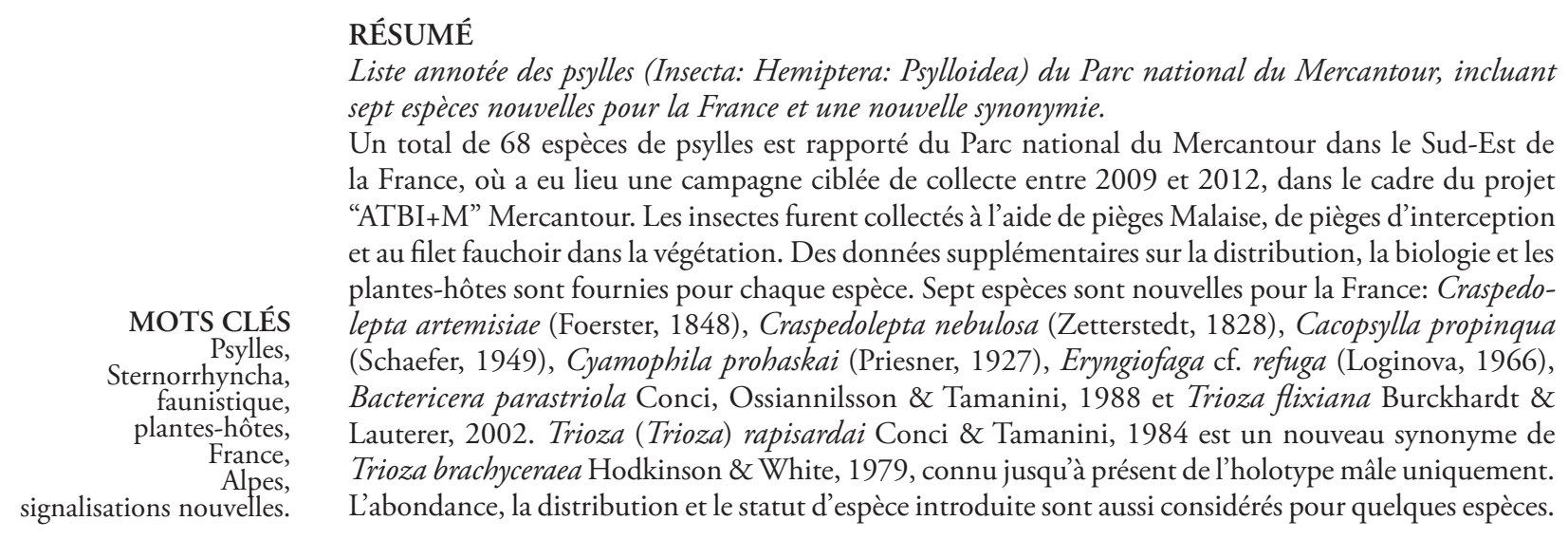

RÉSUMÉ

Liste annotée des psylles (Insecta: Hemiptera: Psylloidea) du Parc national du Mercantour, incluant "ATBI+M" Mercantour. Les insectes furent collectés à l'aide de pièges Malaise, de pièges d'interception et au filet fauchoir dans la végétation. Des données supplémentaires sur la distribution, la biologie et les plantes-hôtes sont fournies pour chaque espèce. Sept espèces sont nouvelles pour la France: Craspedolepta artemisiae (Foerster, 1848), Craspedolepta nebulosa (Zetterstedt, 1828), Cacopsylla propinqua (Schaefer, 1949), Cyamophila prohaskai (Priesner, 1927), Eryngiofaga cf. refuga (Loginova, 1966), Bactericera parastriola Conci, Ossiannilsson \& Tamanini, 1988 et Trioza flixiana Burckhardt \& Trioza brachyceraea Hodkinson \& White, 1979, connu jusqu'à présent de l'holotype mâle uniquement. L'abondance, la distribution et le statut d'espèce introduite sont aussi considérés pour quelques espèces.

\section{INTRODUCTION}

Psylloidea, or jumping plant-lice, are small sap-sucking insects with a world-wide distribution, found on a large variety of plants. Whereas the vast majority of the $c .3900$ described species do not seem to have a major impact on their hostplant, a few are economically important pests on crops or ornamental trees.

From a historical point of view, the oldest citation of an adult psyllid and the first mention of a higher taxonomic rank (despite being from pre-Linnean times) for psyllids was made in France by the entomologist Réaumur in 1737, who observed Homotoma ficus (Linnaeus, 1758) and Psylla buxi (Linnaeus, 1758) in his garden near Paris (Charenton).

The Palaearctic fauna has been thoroughly studied for more than 250 years, and lists have been published for several countries or regional areas, but never for France. Seljak et al. (2008) consider the list of psyllids as nearly complete for Central Europe, but this is far from being true for Western Europe. However, a list of 169 species for France is available from the Fauna Europaea website (Burckhardt 2004), and has served as our reference list for the psyllids of France, with a few exceptions mentioned in the text. Full synonymies are not given here, since all taxonomic information can be found in the Psyl'list online database (Ouvrard 2014).

Nine psyllid species have been previously reported from the Alpes-Maritimes department in previous publications: Camarotoscena speciosa (Flor, 1861) in Cotte (1915), Euphyllura olivina (Costa, 1839) in Balachowsky \& Mesnil (1935) and Peragallo (1881), Livia junci (Schrank, 1789) and Psylla alni (Linnaeus, 1758) in Risso (1826), Psyllopsis fraxini (Linnaeus, 1758) in Béguinot (2002) and Cotte (1915), Trichochermes walkeri (Foerster, 1848), Trioza centranthi (Vallot, 1829) and Trioza kiefferi Giard, 1902 in Béguinot (2002) and Trioza rumicis Löw, 1880 in Cotte (1915).

With the exception of two species in the online ATBI database (http://www.atbi.eu/mercantour-marittime/?q=node/481), namely Calophya rhois (Löw, 1877) and Trioza centranthi (Vallot, 1829), no psyllids have been reported from the
Mercantour National Park before, and only Livilla pyrenaea (Mink, 1859) has been reported from the Alpi Marittime National Park (Conci \& Tamanini 1988). The situation is different for the rest of the Alps, where many species are recorded from the Austrian (Wagner \& Franz 1961), Swiss (Burckhardt 1983; Burckhardt et al. 2007: 47 species from a relatively small area in eastern Switzerland) and Italian (Tamanini 1977; Rapisarda \& Conci 1989; Conci et al. 1993, 1996) parts.

The full list of collected and identified psyllids is given, together with collecting data, a report of the known distribution of each species and comments concerning the available descriptions and elements of the biology (including hostplants) of each species.

\section{MATERIAL AND METHODS}

From 8 June 2009 to 15 October 2010, 697 (288 males and 409 females) psyllid specimens were collected in 92 Malaise traps and 46 interception traps set up in different locations and at two different altitudes (around 1400 and $2000 \mathrm{~m}$ ) in the Mercantour National Park (Deharveng et al. 2015), as part of the project "ATBI+M" Mercantour dedicated to the inventory and monitoring of the whole biodiversity in the area (more information available at: http://www.atbi.eu/ mercantour-marittime/).

All samples collected during the "Terrestrial Invertebrates fieldwork module" of the ATBI Mercantour project were labelled using a coding system where "M" refers to Mercantour, "9" or "10" indicates the sampling year, "BOR", "SES" or "CAI" the sampling area, " $1400 / 1500$ or 2000 " the sampling altitude, T1 to T8 the sequence of the successive sampling periods, "M1 or M2" the Malaise traps and "IN" the interception traps. Detailed information on the trap locations and geographic coordinates, the sampling periods and the main vegetation present at the sampling sites are given in Table 1. The codes and corresponding dates of the successive sampling periods for each sampling site are given in Table 2. 
TABLE 1. - Sample codes and general information on the location of the sampling sites (all in Alpes-Maritimes, 06), their main vegetation and the sampling period.

\begin{tabular}{|c|c|c|c|c|c|c|c|c|c|}
\hline \multirow[b]{2}{*}{ Year } & \multirow[b]{2}{*}{ Sample codes } & \multirow[b]{2}{*}{ Commune } & \multirow[b]{2}{*}{ Locality } & \multirow[b]{2}{*}{ Vegetation } & \multirow[b]{2}{*}{ Altitude } & \multirow[b]{2}{*}{ Longitude } & \multirow[b]{2}{*}{ Latitude } & \multicolumn{2}{|c|}{ Sampling Nb of } \\
\hline & & & & & & & & period & samples \\
\hline 2009 & $\begin{array}{l}\text { M09-BOR1400 } \\
\text { M1 \& IN }\end{array}$ & $\begin{array}{l}\text { St-Martin- } \\
\text { Vésubie }\end{array}$ & Le Boréon & $\begin{array}{l}\text { Meadows and spruce } \\
\text { forest }\end{array}$ & 1540 & 7.2871439 & 44.1146875 & 11.VI-15.X & 8 (T1-T8) \\
\hline 2009 & M09-BOR1400 M2 & $\begin{array}{l}\text { St-Martin- } \\
\text { Vésubie }\end{array}$ & Le Boréon & $\begin{array}{l}\text { Meadows and spruce } \\
\text { forest }\end{array}$ & 1549 & 7.2890533 & 44.1143415 & 11.VI-15.X & 8 (T1-T8) \\
\hline 2009 & M09-BOR2000 M1 & Valdeblore & Col de Salèse & $\begin{array}{l}\text { Meadows, } \\
\text { Rhododendrons, larch } \\
\text { and spruce forest }\end{array}$ & 2058 & 7.23698 & 44.13734 & 10.VI-15.X & 8 (T1-T8) \\
\hline 2009 & M09-BOR2000 M2 & Valdeblore & Col de Salèse & Larch forest & 2032 & 7.2352837 & 44.1388598 & 10.VI-15.X & 8 (T1-T8) \\
\hline 2009 & M09-BOR2000 IN & Valdeblore & Col de Salèse & Larch forest & 2047 & 7.2365699 & 44.1372935 & 10.VI-15.X & 8 (T1-T8) \\
\hline 2009 & M09-SES1400 M1 & $\begin{array}{l}\text { St-Dalmas- } \\
\text { le-Selvage }\end{array}$ & $\begin{array}{l}\text { Vallon de } \\
\text { St-Dalmas: } \\
\text { La Buisse }\end{array}$ & $\begin{array}{l}\text { Meadows, broadleaved } \\
\text { and larch forest }\end{array}$ & 1437 & 6.8875257 & 44.2848357 & 9.VI-15.X & 8 (T1-T8) \\
\hline 2009 & M09-SES1400 M2 & $\begin{array}{l}\text { St-Dalmas- } \\
\text { le-Selvage }\end{array}$ & $\begin{array}{l}\text { Vallon de } \\
\text { St-Dalmas: } \\
\text { La Buisse }\end{array}$ & $\begin{array}{l}\text { Meadows, broadleaved } \\
\text { and larch forest }\end{array}$ & 1421 & 6.8867683 & 44.2850731 & 9.VI-15.X & 8 (T1-T8) \\
\hline 2009 & M09-SES1400 IN & $\begin{array}{l}\text { St-Dalmas- } \\
\text { le-Selvage }\end{array}$ & $\begin{array}{l}\text { Vallon de } \\
\text { St-Dalmas: } \\
\text { La Buisse }\end{array}$ & $\begin{array}{l}\text { Meadows, broadleaved } \\
\text { and larch forest }\end{array}$ & 1436 & 6.8876092 & 44.2847834 & 9.VI-15.X & 8 (T1-T8) \\
\hline 2009 & M09-SES2000 M1 & $\begin{array}{l}\text { St-Dalmas- } \\
\text { le-Selvage }\end{array}$ & $\begin{array}{l}\text { Bois de } \\
\text { Sestrière }\end{array}$ & Larch forest & 1966 & 6.8240421 & 44.2927562 & 8.VI-15.X & 8 (T1-T8) \\
\hline 2009 & M09-SES2000 M2 & $\begin{array}{l}\text { St-Dalmas- } \\
\text { le-Selvage }\end{array}$ & $\begin{array}{l}\text { Bois de } \\
\text { Sestrière }\end{array}$ & Larch forest & 2011 & 6.8228732 & 44.2925219 & 8.VI-15.X & 8 (T1-T8) \\
\hline 2009 & M09-SES2000 IN & $\begin{array}{l}\text { St-Dalmas- } \\
\text { le-Selvage }\end{array}$ & $\begin{array}{l}\text { Bois de } \\
\text { Sestrière }\end{array}$ & Larch forest & 1995 & 6.8235944 & 44.2926706 & 8.VI-15.X & 8 (T1-T8) \\
\hline 2010 & $\begin{array}{l}\text { M10-CAI1400 } \\
\text { M1 \& IN }\end{array}$ & Saorge & $\begin{array}{l}\text { Forêt de } \\
\text { Caïros: } \\
\text { Vallerasse }\end{array}$ & Fir forest & 1379 & 7.45615 & 44.00338 & 1.VII-15.X & 7 (T1-T7) \\
\hline 2010 & M10-CAl1400 M2 & Saorge & $\begin{array}{l}\text { Forêt de } \\
\text { Caïros: } \\
\text { Vallerasse }\end{array}$ & Fir forest & 1387 & 7.45692 & 44.00343 & 1.VII-15.X & 7 (T1-T7) \\
\hline 2010 & M10-CAl1400 IN & Saorge & $\begin{array}{l}\text { Forêt de } \\
\text { Caïros: } \\
\text { Vallerasse }\end{array}$ & Fir forest & 1398 & 7.45577 & 44.00344 & 1.VII-15.X & 7 (T1-T7) \\
\hline 2010 & M10-CAI2000 M1 & Saorge & $\begin{array}{l}\text { Tête de la } \\
\text { Poudrière }\end{array}$ & Larch forest & 1953 & 7.42407 & 44.01454 & 30.VI-15.X & 7 (T1-T7) \\
\hline 2010 & M10-CAI2000 M2 & Saorge & $\begin{array}{l}\text { Tête de la } \\
\text { Poudrière }\end{array}$ & Larch forest & 1992 & 7.42459 & 44.01388 & 30.VI-15.X & 7 (T1-T7) \\
\hline 2010 & M10-CAI2000 IN & Saorge & $\begin{array}{l}\text { Tête de la } \\
\text { Poudrière }\end{array}$ & Larch forest & 1985 & 7.42467 & 44.01400 & 30.VI-15.X & 7 (T1-T7) \\
\hline
\end{tabular}

TABLE 2. - Codes and dates of the successive sampling periods for each sampling site.

\begin{tabular}{|c|c|c|c|c|c|c|c|c|c|c|}
\hline Year & Site & T1 & T2 & T3 & T4 & T5 & T6 & T7 & T8 & T9 \\
\hline 2009 & $\mathrm{OP}$ & 11.VI & 24.1 & VII & 21. & 1 & $27 . \mathrm{V}$ & 18.1 & $5 . x$ & 政 \\
\hline 2009 & BOR & $10 . V$ & $24 . V$ & 4.VII & $24 . V$ & 13. VII & $27 . \mathrm{V}$ & $X$ & $x$ & ple \\
\hline 2009 & SES1400 & 9.VI-30.VI & 30.VI-10.VII & 10.VII-23.VII & 23.VII-7.VIII & 7.VIII-19.VIII & 19.VIII-22.IX & 22.IX-7.X & $5 . X$ & nple \\
\hline 2009 & SES2000 & 8.VI-24.VI & 24.VI-10.VII & 10.VII-23.VII & 23.VII-7.VIII & 7.VIII-19.VIII & 19.VIII-22.IX & 22.IX-7.X & 7.X- & no \\
\hline 2010 & CAl1 & 1.VII-1 & 16.VII- & 31.VII-16.VIII & 16.VIII-30.VIII & 30.VIII-15.IX & 15.IX & $30.1 X-15 . X$ & no sample & no sample \\
\hline 2010 & CAI2000 & 30.VI-16.VII & 16.VII-31.VII & 31.VII-16.VIII & 16.VIII-30.VIII & 30.VIII-15.IX & 15.IX-30.IX & $30.1 X-15 . X$ & no sample & no sample \\
\hline
\end{tabular}

In addition, the third author (CC) has made several collecting trips to the Mercantour National Park (June 1994, 2 and 4 July 2008; 9, 13, 16-23 July 2009; 8-15 July 2011). A few specimens collected in 2012 by Jean-Claude Streito (INRA, Montpellier) are also reported, as well as specimens collected by Igor Malenovský (Moravian Museum, Brno, Czech Republic) between 1994 and 1996. All specimens were caught by sweeping low vegetation or beating branches of bushes and trees.
In addition to the reference list of Fauna Europaea, the present data have been cross-checked with the dataset of the "Inventaire National du Patrimoine Naturel" (INPN: http:// inpn.mnhn.fr/accueil/index) for the completeness of data from France, and with both the GBIF portal (http://data. gbif.org) and the Psyl'list database (Ouvrard 2014) for the world-wide distribution.

The higher classification follows Burckhardt \& Ouvrard (2012). 
The Plant List (http://www.theplantlist.org/) has been used as the reference for the plant scientific names.

\section{ABBREVIATIONS}

\section{Institutions}

BMNH Natural History Museum, London;

CBGP Centre de biologie pour la Gestion des Populations, Montpellier;

MHNG Muséum d'Histoire naturelle de Genève;

MMBC Moravian Museum, Brno, Czech Republic;

MNHN Muséum national d'Histoire naturelle, Paris;

NHMB Naturhistorisches Museum, Basel.

\section{SYSTEMATICS}

Superfamily PSYLLOIDEA Latreille, 1807

Family APHALARIDAE Löw, 1879

Subfamily APHALARINAE Löw, 1879

Genus Aphalara Foerster, 1848

Aphalara sauteri Burckhardt, 1983

Aphalara sauteri Burckhardt, 1983: 47.

Material EXAmined. - France. Alpes-de-Haute-Provence, Fours, Bois de la Tellière, $1420 \mathrm{~m}, 4^{\circ} 19^{\prime}$ '5.415"N-637'52.8996”'E, 01.IX.2012 C. Cocquempot (CBGP), 1 adult; Alpes-Maritimes, Bolène-Vesubie, massif de la cime du Diable, 1800-2600 m, 28.VIII.1995, $10^{7}$ I. Malenovský (MMBC); Alpes-Maritimes, La Brigue, French-Italian border between Mt Grai (2013 m) and Cime de Marte (2135 m), 21.VIII.1996, 1, I. Malenovský (MMBC).

Distribution. - Fauna Europaea: Austria, France, Italy (mainland + Sardinia), Slovakia, Spain, Switzerland; INPN: no data; additional data from Psyl'list: Slovenia.

\section{COMMENTS}

Description and biology in Burckhardt \& Lauterer (1997). Host-plant: Rumex scutatus L. (Polygonaceae).

Genus Craspedolepta Enderlein, 1921

\section{Craspedolepta artemisiae (Foerster, 1848)}

Aphalara artemisiae Foerster, 1848: 96.

Material EXAmined. - France. Alpes-de-Haute-Provence, Fours,

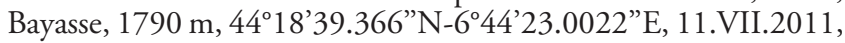
on Artemisia sp., C. Cocquempot (CBGP), 102 adults.

Distribution. - Fauna Europaea: Bulgaria, Czech Republic, Germany, Moldova, Poland, Romania, Russia Central, Slovakia, Spain (doubtful), Ukraine; INPN: no data; additional data from Psyl'list: Hungary.

\section{COMMENTS}

Description in Wagner (1947), Vondráček (1957), Loginova (1963), Klimaszewski (1983) and Konovalova (1988). Biology unknown. Host-plant: Artemisia campestris L. (Asteraceae). New for France.
Craspedolepta malachitica (Dahlbom, 1851)

Psylla malachitica Dahlbom, 1851: 177.

Material EXAmined. - France. Alpes-Maritimes, Saint-Dalmasde-Tende, Refuge Neige et Merveilles, $1545 \mathrm{~m}, 44^{\circ} 4{ }^{\prime} 12.0318^{\prime \prime N}$ 7³0'44.064"E, 17.VII.2009, on Artemisia sp. C. Cocquempot (CBGP), 43 adults; Alpes-Maritimes, Tende, Les Mesches, $1450 \mathrm{~m}$, 10.VII.1994, 140", 5o , I. Malenovský (MMBC).

Distribution. - Fauna Europaea: Austria, Czech Republic, Finland, France, Germany, Great Britain, Italy, Moldova, Norway, Poland, Romania, Russia, Slovakia, Sweden, Switzerland, Ukraine; INPN: no data; additional data from Psyl'list: Armenia, Georgia, Kazakhstan, Mongolia.

\section{COMMENTS}

Description in Hodkinson \& White (1979a) and Ossiannilsson (1992). Biology in Conci et al. (1993) and Ossiannilsson (1992). Host-plants: Artemisia absinthium L. and A. maritima Ledeb. (Asteraceae).

\section{Craspedolepta nebulosa (Zetterstedt, 1828)}

Chermes nebulosa Zetterstedt, 1828: 551.

Material eXAmined. - France. Alpes-Maritimes, Lantosque, Col d'Andrion (Col du Fort), 1990 m, 44²1'3.8202"N-7¹5'12.1206”E, 13.VII.2009, on Epilobium angustifolium L., C. Cocquempot (CBGP), 48 adults.

Distribution. - Fauna Europaea: Austria, Czech Republic, Denmark, Finland, Germany, Great Britain, Italy, Moldova, Norway, Poland, Romania, Russia, Slovakia, Sweden, Switzerland, Ukraine; INPN: no data; additional data from Psyl'list: Canada, Ireland, Kazakhstan, United States of America (Alaska, California, Colorado, New Hampshire, Washington).

\section{COMMENTS}

Description in Hodkinson \& White (1979a) and Ossiannilsson (1992). Biology in Ossiannilsson (1992), Conci et al. (1993) and Lauterer (1993). Host-plant: Epilobium angustifolium L. (Onagraceae). New for France.

\section{Craspedolepta nervosa (Foerster, 1848)}

Aphalara nervosa Foerster, 1848: 90.

Material eXAmined. - France. Alpes-Maritimes, Saint-MartinVésubie, M09-BOR1400-T2-M2, $10^{7}$ (BMNH); same locality, M09-BOR1400-T1-M1, 2 \% (MNHN); Alpes-Maritimes, SaintDalmas-le-Selvage, M09-SES1400-T1-IN, 1 ㅇ (BMNH); same locality, M09-SES1400-T1-M1, $10^{7}$ (MNHN); Alpes-de-HauteProvence, Larche, Vallon du Lauzanier, val Fourane, 1923 m,

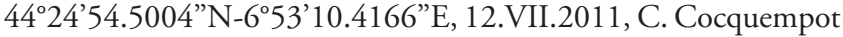
(CBGP), 6 adults; Alpes-Maritimes, Moulinet, Col de Turini, piste de Gasta-Fume, 1625 m, 4358'27.1482”N-7²3'42.7374”E, 02.VII.2008, C. Cocquempot (CBGP), 18 adults.

Distribution. - Fauna Europaea: Austria, Belgium, Czech Republic, Denmark, Finland, France, Germany, Great Britain, Ireland, Italy, Moldova, Norway, Poland, Romania, Russia, Slovakia, Sweden, 
Switzerland, The Netherlands, Ukraine; INPN: no data; additional data from Psyl'list: Hungary, Iraq, Mongolia, Slovenia.

\section{COMments}

Description in Hodkinson \& White (1979a) and Ossiannilsson (1992). Biology in Conci et al. (1993). Host-plants: several species of Achillea (Asteraceae).

\section{Subfamily RHINOCOLINAE Vondráček, 1957 Genus Lisronia Loginova, 1976}

Lisronia varicicosta (Hodkinson \& Hollis, 1981)

Rhachistoneura varicicosta Hodkinson \& Hollis, 1981: 66.

Material eXamined. - France. Alpes-Maritimes, Saorge, M10CAI2000-T1-M2, 1 우 (MNHN).

DisTRIBUTION. - Fauna Europaea: France, Greece, Italy (mainland + Sardinia + Sicily), Portugal, Spain (Balearic Is.), North Africa; INPN: no data; additional data from Psyl'list: Algeria, Israel.

\section{COMMENTS}

Description in Hodkinson \& Hollis (1981) and Rapisarda (1987). Biology in Conci et al. (1993). Host-plants: several species of Cistus and Tuberana lignosa (Sweet) Samp. (Cistaceae).

\section{Genus Rhinocola Foerster, 1848}

Rhinocola aceris (Linnaeus, 1758)

Chermes aceris Linnaeus, 1758: 454.

Material examined. - France. Alpes-Maritimes, SaintDalmas-le-Selvage, M09-SES1400-T2-M2, $10^{\prime \prime}$ (MNHN); Alpes-de-Haute-Provence, Entraunes, Oustamura, $1360 \mathrm{~m}$,

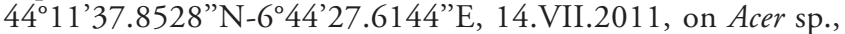
C. Cocquempot (CBGP), 2 adults; Alpes-de-Haute-Provence, Entraunes, Piste de la Cascade de l'Aiglière, 1420 m, 44²'124.9732"N64433.4782”E, 14.VII.2011, on Acer sp., C. Cocquempot (CBGP), 6 adults; Alpes-de-Haute-Provence, Colmars-les-Alpes, Piste de Ratéry, 1670 m, 44¹1'16.5804”N-6³9'15.4548”E, 10.VII.2011, on Acer sp., C. Cocquempot (CBGP), 1 adult; Alpes-Maritimes, Saint-Dalmas-de-Tende, Refuge Neige et Merveilles, $1545 \mathrm{~m}$,

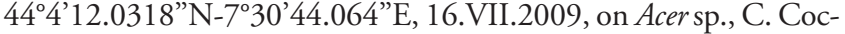
quempot (CBGP), 53 adults.

Distribution. - Fauna Europaea: Austria, Belgium, Bulgaria, Czech Republic, Denmark, Finland, France, Germany, Great Britain, Greece, Italy (mainland + Sicily), Moldova, Norway, Poland, Romania, Russia, Slovakia, Slovenia, Sweden, Switzerland, Ukraine; INPN: no data; additional data from Psyl'list: Armenia, Baltic States, Caucasus, Georgia, Hungary, Tunisia, Turkey.

\section{COMMENTS}

Description in Hodkinson \& White (1979a) and Ossiannilsson (1992). Biology in Conci et al. (1993) and Lauterer (2011). Host-plants: several species of Acer (Aceraceae).
Subfamily SPONDYLiASPIDINAE Schwarz, 1898

Genus Ctenarytaina Ferris \& Klyver, 1932

Ctenarytaina eucalypti (Maskell, 1890)

Rhinocola eucalypti Maskell, 1890: 160.

Material eXamined. - France. Alpes-Maritimes, Saint-Dalmasle-Selvage, M09-SES1400-T1-M2, $20^{\circ}$ (MNHN, BMNH).

Distribution. - Fauna Europaea: France, Germany, Great Britain, Ireland, Italy, Portugal (mainland + Madeira), Spain (mainland + Canary Is.), Switzerland, Afro-tropical region, Australian region, Neotropical region, Oriental region; INPN: no data; additional data from Psyl'list: Australia, Bolivia, Brazil, Chile, Colombia, New Zealand, Papua New Guinea, Portugal (Azores), South Africa, Sri Lanka, United States of America (California).

\section{COMMENTS}

Description in Hodkinson \& White (1979a). Biology in Burckhardt (1994a, 1998). Biological control review in Queiroz et al. (2012). Native to Australia, including Tasmania. Reported from France as early as 1925 (Mercier \& Poisson 1926). Host-plants: several species of Eucalyptus (Myrtaceae).

Family CALOPHYIDAE Vondráček, 1957

Subfamily CALOPHYINAE Vondráček, 1957

Genus Calophya Löw, 1879

Calophya rhois (Löw, 1877)

Psylla rhois Löw, 1877: 148.

Material eXAmined. - France. Alpes-Maritimes, Saorge, Route du Caïros, 604 m, 4359'49.1064”N-7³0'22.9602”E, 20.VII.2009, on Rhus cotinus L. [= Cotinus coggygria Scop.], C. Cocquempot (CBGP), 54 adults.

Distribution. - Fauna Europaea: Austria, Bulgaria, Czech Republic, France, Great Britain, Greece, Italy, Moldova, Romania, Slovenia, Spain, Ukraine. East Palaearctic, Near East; INPN: observed in 2009 in Mercantour National Parc (data ATBI Mercantour; square $10 \mathrm{~km}$ L93E106N633); additional data from Psyl'list: China, Georgia, Hungary, Russia, Serbia, Turkey.

\section{COMMENTS}

Description in Hodkinson \& White (1979a). Biology in Conci et al. (1996). Host-plant: Cotinus coggygria Scop. (Anacardiaceae).

\section{Family LIVIIDAE Löw, 1879}

Subfamily EUPHYLLURINAE Crawford, 1914

Genus Psyllopsis Löw, 1879

Psyllopsis fraxini (Linnaeus, 1758)

Chermes fraxini Linnaeus, 1758: 454.

MATERIAl EXAminED. - France. Alpes-de-Haute-Provence, Colmarsles-Alpes, 1350 m, 4411'12.8472"N-638'4.6896”E, 10.VII.2011, on Fraxinus sp., C. Cocquempot (CBGP), 1 adult; Alpes-Maritimes, Saorge, L'Abéon, 1266 m, 4359'39.3288”N-7²9'14.355”E, 
20.VII.2009, on Fraxinus sp., C. Cocquempot (CBGP), 16 adults; Alpes-de-Haute-Provence, Entraunes, Piste de la Cascade de l'Aiglière, 1420 m, 44¹2'24.9732"N-644'33.4782"E, 14.VII.2011, on Fraxinus sp., C. Cocquempot (CBGP), 1 adult; Alpes-de-Haute-Provence, Entraunes, Le Clôt, 1324 m, 44¹1'47.0724”N-644'40.9878”E, 11.VII.2011, C. Cocquempot (CBGP), 1 adult.

Distribution. - Fauna Europaea: Austria, Belgium, Czech Republic, Denmark, Finland, France, Germany, Great Britain, Ireland, Italy (mainland + Sicily), Moldova, Norway, Poland, Romania, Russia, Slovakia, Slovenia, Spain, Sweden, Switzerland, The Netherlands, Ukraine; INPN: no data; additional data from Psyl'list: Australia (Tasmania), Hungary, Iran, New Zealand, Slovenia, United States of America (New York), West Himalaya (Uttaranchal = Uttarakhand).

\section{COMMENTS}

Description in Hodkinson \& White (1979a), Conci \& Tamanini (1990) and Ossiannilsson (1992). Biology in Ossiannilsson (1992) and Conci et al. (1993). Host-plants: several species of Fraxinus (Oleaceae).

\section{Psyllopsis fraxinicola (Foerster, 1848)}

\section{Psylla fraxinicola Foerster, 1848: 73.}

Material EXAMINED. - France. Alpes-Maritimes, Saint-Dalmas-leSelvage, M09-SES1400-T2-M2, $10^{7}$ (MNHN); Alpes-Maritimes, Saint-Dalmas-de-Tende, Refuge Neige et Merveilles, $1545 \mathrm{~m}$,

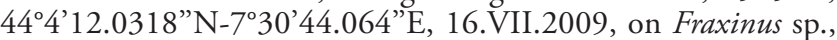
C. Cocquempot (CBGP), 49 adults; Alpes-Maritimes, Saorge,

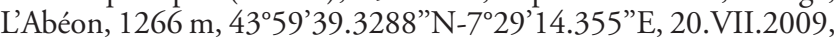
on Fraxinus sp., C. Cocquempot (CBGP), 81 adults; Alpes-de-HauteProvence, Entraunes, Piste de la Cascade de l'Aiglière, $1420 \mathrm{~m}$, $44^{\circ} 12^{\prime 2} 24.9732$ "N-644'33.4782"E, 14.VII.2011, on Fraxinus sp., C. Cocquempot (CBGP), 2 adults.

Distribution. - Fauna Europaea: Austria, Belgium, Czech Republic, Denmark, Finland, France, Germany, Great Britain, Greece, Ireland, Italy (mainland + Sardinia + Sicily), Malta, Moldova, Norway, Poland, Romania, Slovakia, Spain, Sweden, Switzerland, The Netherlands, Ukraine; INPN: no data; additional data from Psyl'list: Algeria, Armenia, Australia, Canada (British Columbia, Nova Scotia), Georgia, Hungary, Kazakhstan, Lithuania, New Zealand, Slovenia, Turkey, United States of America (California, Connecticut, Idaho, New Jersey, Utah, Washington DC).

\section{COMMENTS}

Description in Hodkinson \& White (1979a), Conci \& Tamanini (1990a) and Ossiannilsson (1992). Biology in Ossiannilsson (1992) and Conci et al. (1993). Host-plants: Fraxinus dipetala Hook. \& Arn. (North America), Fraxinus excelsior L. and Fraxinus ornus L. (Oleaceae).

Family PSYLLIDAE Latreille, 1807

Subfamily ACIZZIINAE White \& Hodkinson, 1985

Genus Acizzia Heslop-Harrison, 1961

Acizzia uncatoides (Ferris \& Klyver, 1932)

Psylla uncatoides Ferris \& Klyver, 1932: 53.

Material EXAmined. - France. Alpes-Maritimes, Saint-Dalmasle-Selvage, M09-SES1400-T2-M2, 1 (MNHN); Alpes-Maritimes,
Saint-Dalmas-de-Tende, Lac de la Muta, 2365 m, 44²'57.2742"N7²6'5.694”'E, 18.VII.2009, C. Cocquempot (CBGP), 12 adults; Alpes-Maritimes, Saint-Dalmas-de-Tende, Lac de Trem, 2270 m,

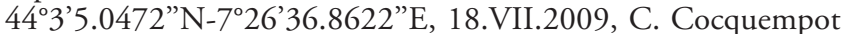
(CBGP), 8 adults; Breil-sur-Roya, Authion, vacherie de l'Ortiguier, $1960 \mathrm{~m}, 43^{\circ} 59^{\prime} 40.6284$ "N-7 26'1.0536"E, on Larix decidua, 20.VII.2009, C. Cocquempot (CBGP), 1 adult; Alpes-Maritimes, Saorge, Forêt de Caïros, M10-CAI2000-T1-M2, $10^{7}$ (MNHN); Alpes-Maritimes, La Brigue, French-Italian border between $\mathrm{Mt}$ Grai $(2013 \mathrm{~m})$ and Cime de Marte (2135 m), 21.VIII.1996, 70", 3요, I. Malenovský (MMBC).

Distribution. - Fauna Europaea: France, Italy (mainland + Sicily), Malta, Portugal (mainland + Azores), Spain (Canary Islands), ex-Yugoslavia; INPN: no data; additional data from Psyl'list: Algeria, Australia, Chile, Colombia, France (Guadeloupe), Great Britain, Israel, Lebanon, Mexico, Montenegro, New Zealand, United States of America (California, Hawaii).

\section{COMMENTs}

Description in Hodkinson \& Hollis (1987). Introduced and established in France, reported for the first time in 1976 (Bain et al. 1976). Native to Australia. Host-plants are species of Acacia and Albizia (Fabaceae). In France, it has been collected on Acacia baileyana F. Muell. Populations of $A$. uncatoides undergo 6 to 8 generations per year in Italy (Conci et al. 1996).

Subfamily PSYLLINAE Latreille, 1807

Genus Baeopelma Enderlein, 1926

Baeopelma foersteri (Flor, 1861)

Psylla foersteri Flor, 1861: 458.

Material eXamined. - France. Alpes-Maritimes, Lantosque, SaintColomban, Camari, 897 m, 4357’7.9956”N-7²0'52.5906”E, 04.VII.2008, on Alnus sp., C. Cocquempot (CBGP), 3 adults.

Distribution. - Fauna Europaea: Austria, Belgium, Bulgaria, Czech Republic, Denmark, Finland, France, Germany, Great Britain, Greece, Ireland, Italy (mainland + Sardinia + Sicily), Moldova, Norway, Poland, Romania, Russia, Slovakia, Slovenia, Spain, Sweden, Switzerland, The Netherlands, Ukraine; INPN: no data; additional data from Psyl'list: Algeria, Caucasus, Hungary, Lebanon, Turkey.

\section{COMMENTS}

Description in Hodkinson \& White (1979a) and Ossiannilsson (1992). Biology in Ossiannilsson (1992) and Conci et al. (1993). Host-plants: Alnus incana (L.) Moench and A. glutinosa (L.) Gaertn. (Betulaceae).

\section{Genus Cacopsylla Ossiannilsson, 1970}

Cacopsylla albipes (Flor, 1861)

Psylla albipes Flor, 1861: 364

Material examined. - France. Alpes-Maritimes, Saorge, M10CAI1400-T5-M2, 1 9 (MNHN); same mocality, M10-CAI2000-T1M1, 1 ㅇ (BMNH); Alpes-Maritimes, Bolène-Vesubie, massif de la cime du Diable, 1800-2600 m, 28.VIII.1995, 10", I. Malenovský (MMBC). 
Distribution. — Fauna Europaea: Austria, France, Germany, Great Britain, Italy, Poland, Slovakia, Switzerland, Ukraine; INPN: no data; additional data from Psyl'list: Bulgaria, Crimea, Czech Republic, Georgia, Slovenia, Turkey.

\section{COMMENTS}

Description in Hodkinson \& White (1979a), Vondráček (1957), Burckhardt (2010). Biology in Conci et al. (1993). Host-plants: several species of Sorbus (Rosaceae).

\section{Cacopsylla ambigua (Foerster, 1848)}

Psylla ambigua Foerster, 1848: 74.

Material eXamined. - France. Alpes-Maritimes, Saint-MartinVésubie, M09-BOR1400-T1-M2, 1 9 (MNHN); Alpes-Maritimes, Valdeblore, M09-BOR2000-T2-M1, 2 (MNHN, BMNH); Alpesde-Haute-Provence, Larche, Malboisset, 1700 m, 44⒉ $42.5298^{\prime \prime} \mathrm{N}$ 651'26.5752"E, 12.VII.2011, on Salix sp., C. Cocquempot (CBGP), 33 adults; Alpes-Maritimes, Castérino, Pont des Gasis, 1540 m, 44 5'19.8276”N-7³0'59.76”'E, 22.VII.2009, on Salix sp., C. Cocquempot (CBGP), 28 adults; Alpes-Maritimes, Lantosque,

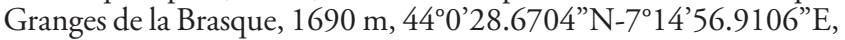
09.VII.2009, on Salix sp., C. Cocquempot (CBGP), 28 adults; Alpes-Maritimes, Lantosque, Saint-Colomban, Camari, 897 m, $43^{\circ} 57^{\prime} 7.9956^{\prime \prime} \mathrm{N}-7^{\circ} 20^{\prime} 52.5906^{\prime \prime} \mathrm{E}, 04 . \mathrm{VII} .2008$, on Salix sp., C. Cocquempot (CBGP), 7 adults; Alpes-Maritimes, Tende, Les Mesches, 1450 m, 10.VII.1994, 1 ơ, 29, I. Malenovský (MMBC).

Distribution. - Fauna Europaea: Austria, Belgium, Czech Republic, Denmark, Finland, France, Germany, Great Britain, Greece, Ireland, Italy (mainland + Sardinia + Sicily), Moldova, Norway, Poland, Romania, Russia, Slovakia, Spain, Sweden, Switzerland, Ukraine; INPN: no data; additional data from Psyl'list: Greenland, Hungary, Japan, Slovenia.

\section{COMMENTS}

Description in Hodkinson \& White (1979a). Biology in Lauterer (1976) and Conci et al. (1993). Host-plants: several species of Salix (Salicaceae).

\section{Cacopsylla breviantennata (Flor, 1861)}

Psylla breviantennata Flor, 1861: 375.

Material examined. - France. Alpes-Maritimes, Saint-Dalmas-le-Selvage, M09-SES1400-T2-M2, 1 ㅇ (MNHN); same locality, M09-SES1400-T8-IN, 1 ( Saorge, M10-CAI1400-T6-M1, $10^{7}$ (MNHN); same locality, M10-CAI2000-T7-M1, $10^{7}$ (MNHN); Alpes-Maritimes, SaintMartin-Vésubie, M09-BOR1400-T1-M1, $10^{\top}$ (BMNH).

Distribution. - Fauna Europaea: Austria, Baleares, Czech Republic, France, Germany, Italy, Romania, Switzerland; INPN: no data; additional data from Psyl'list: Greenland, Hungary, Japan, Slovenia.

\section{COMMENTS}

Description in Vondráček (1957), Dobreanu \& Manolache (1962), Klimaszewski (1963) and Burckhardt (2010). Biology in Conci et al. (1993). Host-plants: Sorbus aria Crantz (Rosaceae).
Cacopsylla brunneipennis (Edwards, 1896)

Psylla brunneipennis Edwards, 1896: 238.

Material eXamined. - France. Alpes-Maritimes, Saint-Martin-Vésubie, M09-BOR1400-T1-M1, 3 9 (MNHN); same locality, M09-BOR1400-T2-M1, 2 \% (MNHN); same locality, M09-BOR1400-T8-M1, 1 ( (MNHN); same locality, M09BOR1400-T1-M2, 4 ㅇ (MNHN); same locality, M09-BOR1400-T2M2, 39 (MNHN); same locality, M09-BOR1400-T8-M2, 1 \% (MNHN); Alpes-Maritimes, Valdeblore, M09-BOR2000-T2-M1, 1 우 (MNHN); same locality, M09-BOR2000-T7-M1, $10^{\prime \prime}$ (MNHN); same locality, M09-BOR2000-T2-M2, 20", 19 (MNHN); AlpesMaritimes, Saint-Dalmas-le-Selvage, M09-SES2000-T1-IN, 1 \% (MNHN); same locality, M09-SES1400-T1-M2, 1 \% (MNHN); same locality, M09-SES1400-T1-M2, 19 (MNHN); Alpes-Maritimes, Saorge, M10-CAI1400-T1-M2, 1 ( $(\mathrm{MNHN})$; same locality, M10-CAI1400-T6-M2, $10^{7}, 1$ ( $(\mathrm{MNHN})$; same locality, M10CAI1400-T3-M2, $10^{7}$ (MNHN); same locality, M10-CAI1400-T7M2, 2 ㅇ (MNHN); same locality, M10-CAI1400-T3-M1, 1 우 (MNHN); same locality, M10-CAI1400-T7-M1, 2 (MNHN); same locality, M10-CAI2000-T1-M1, 707, 36\% (MNHN); same locality, M10-CAI2000-T2-M1, 1 ( $(\mathrm{MNHN})$; same locality, M10-CAI2000-T3-M1, $50^{7}, 24$ 으 (MNHN); same locality, M10CAI2000-T4-M1, 8 \% (MNHN); same locality, M10-CAI2000-T5M1, 6우 ; same locality, M10-CAI2000-T6-M2, $10^{\prime \prime}$, 7 우 (MNHN); same locality, M10-CAI2000-T7-M1, 307, 18ㅇ (MNHN); same locality, M10-CAI2000-T1-M2, 30", $24 \%$ (MNHN); same locality, M10-CAI2000-T2-M2, $10^{7}, 3$ ㅇ (MNHN); same locality, M10-CAI2000-T3-M2, $10^{\prime \prime}$, 5 ㅇ (MNHN); same locality, M10CAI2000-T3-IN, 1 우 (MNHN); same locality, M10-CAI2000-T1M2, 1 ㅇ (MNHN); same locality, M10-CAI2000-T7-M2, 20", 3 \% (BMNH); Alpes-Maritimes, Breil-sur-Roya, Authion, vacherie de l'Ortiguier, $1960 \mathrm{~m}, 43^{\circ} 59^{\prime} 40.6284^{\prime} \mathrm{N}-7^{\circ} 26^{\prime} 1.0536$ ” E, 20.VII.2009, on Larix decidua Mill., C. Cocquempot (CBGP), 62 adults; same data except Moulinet, Col de Turini, $1610 \mathrm{~m}$, 4358'37.56"N-7 23'30.7782”'E, 09.VII.2009, on herbaceous plants, 1 adult; same data except Lantosque, Piste du col du Fort, 1770 m, $44^{\circ} 0^{\prime} 43.9668^{\prime \prime} \mathrm{N}-7^{\circ} 14^{\prime} 45.3546$ "E, 13.VII.2009, on Larix decidua Mill., 21 adults; same data except Col d'Andrion (Col du Fort),

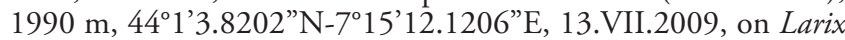
decidua Mill., 1 adult; same data except Lantosque, Granges de la

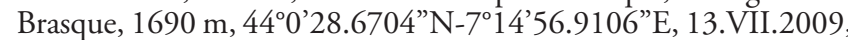
1 adult; same data except Saint-Dalmas-de-Tende, Pas de La Nauque, 2130 m, 44³'6.4722"N-7³1'21.5148”'E, 17.VII.2009, on Larix decidua Mill., 8 adults; same data except Lac de Trem, 2270 m, $44^{\circ} 3^{\prime} 5.0472^{\prime \prime N}-7^{\circ} 26^{\prime} 36.8622^{\prime \prime E}$, 18.VII.2009, 4 adults; AlpesMaritimes, Tende, Les Mesches, 1450 m, 10.VII.1994, $20^{\text {", }}$ 2웅 I. Malenovský (MMBC); Alpes-Maritimes, Breil-sur-Roya, Authion, 1850 m, 28.VIII.1995, $10^{\text {* }}$, I. Malenovský (MMBC); Alpes-Maritimes, Bolène-Vesubie, massif de la cime du Diable, 1800-2600 m, 28.VIII.1995, 90", 21 ㅇ, I. Malenovský (MMBC); Alpes-Maritimes, La Brigue, French-Italian border between Mt Grai $(2013 \mathrm{~m})$ and Cime de Marte (2135 m), 21.VIII.1996, 10 ơ, 11 \&, I. Malenovský (MMBC).

DistribUtion. - Fauna Europaea: Austria, Bulgaria, Czech Republic, Denmark, Finland, France, Germany, Great Britain, Ireland, Italy (mainland + Sardinia), Norway, Poland, Romania, Russia, Slovakia, Spain, Sweden, Switzerland, Ukraine; INPN: no data; additional data from Psyl'list: Caucasus, Hungary, Slovenia.

\section{COMMENTS}

Description in Hodkinson \& White (1979) and Ossiannilsson (1992). Biology in Conci et al. (1996). Host-plants: several species of Salix (Salicaceae). 


\section{Cacopsylla crataegi (Schrank, 1801)}

Chermes crataegi Schrank, 1801: 142.

Material EXAMINED. - France. Alpes-Maritimes, Breil-sur-Roya, Authion, vacherie de l'Ortiguier, $1960 \mathrm{~m}, 43^{\circ} 59^{\prime} 40.6284$ "N$7^{\circ} 26^{\prime} 1.0536$ ”E, 20.VII.2009, on Larix decidua Mill., C. Cocquempot (CBGP), 1 adult.

Distribution. - Fauna Europaea: Austria, Belgium, Bulgaria, Czech Republic, Denmark, France, Germany, Great Britain, Ireland, Italian (mainland + Sardinia + Sicily), Moldova, Poland, Romania, Slovakia, Spain, Sweden, Switzerland, Ukraine; INPN: no data; additional data from Psyl'list: Algeria, Caucasus, Georgia, Greece, Hungary, India, Iran, Israel, Morocco, Slovenia.

\section{COMMENTS}

Description in Hodkinson \& White (1979a), Ossiannilsson (1992) and Burckhardt (2010). Biology in Ossiannilsson (1992) and Conci et al. (1993). Host-plants: several species of Crataegus (Rosaceae).

\section{Cacopsylla iteophila (Löw, 1876)}

Psylla iteophila Löw, 1876: 196.

Material EXAMined. — France. Alpes-de-Haute-Provence, Colmarsles-Alpes, Col des Champs, 1920 m, 44¹1'4.884"N- 640'15.3984"E, 10.VII.2011, on Larix decidua Mill., C. Cocquempot (CBGP), 8 adults.

Distribution. - Fauna Europaea: Austria, France, Italy, Poland, Slovakia, Spain, Switzerland; INPN: no data; additional data from Psyl'list: Slovenia.

\section{COMments}

Description in Conci \& Tamanini (1990b). Biology in Conci et al. (1993). Host-plants: several species of Salix (Salicaceae).

\section{Cacopsylla limbata (Meyer-Dür, 1871)}

Psylla limbata Meyer-Dür, 1871: 392.

Material eXAmined. - France. Alpes-Maritimes, La Brigue, French-Italian border between Mt Grai $(2013 \mathrm{~m})$ and Cime de Marte (2135 m), 21.VIII.1996, 10", I. Malenovský (MMBC).

Distribution. - Fauna Europaea: France, Italy, Switzerland; INPN: no data; additional data from Psyl'list: no additional data.

\section{COMMENTS}

Description in Conci \& Tamanini (1982). Biology in Conci \& Tamanini (1989b) and Conci et al. (1993). Host-plants: Rhamnus alpina L. and R. pumila Turra (Rhamnaceae).

\section{Cacopsylla melanoneura (Foerster, 1848)}

Psylla melanoneura Foerster, 1848: 75.

Material eXamined. - France. Alpes-Maritimes, Valdeblore, M09-BOR2000-T2-M1, $20^{7}$ (MNHN); same locality, M09BOR2000-T2-M2; Alpes-Maritimes, Saint-Dalmas-le-Selvage,
M09-SES2000-T2-M1, 3 (MNHN); Alpes-Maritimes, Saorge, M10CAI1400-T3-M1, $10^{7}$ (MNHN); same locality, M10-CAI1400-T3M2, 1 ㅇ (MNHN); same locality, M10-CAI1400-T3-M1, $10^{\prime \prime}, 1$ 우 (BMNH); same locality, M10-CAI1400-T4-M1, $10^{7}$ (MNHN); Alpes-Maritimes, Saint-Dalmas-de-Tende, Pas de La Nauque, 2130 m, 44³'6.4722"N-7³1'21.5148"E, 17.VII.2009, on Larix decidua Mill., C. Cocquempot (CBGP), 1 adult; Alpes-Maritimes, La Brigue, French-Italian border between Mt Grai $(2013 \mathrm{~m})$ and Cime de Marte (2135 m), 21.VIII.1996, 60", 2\%, I. Malenovský (MMBC).

Distribution. - Fauna Europaea: Austria, Belgium, Bulgaria, Channel Is., Croatia, Czech Republic, Danish mainland, French mainland, Germany, Great Britain, Greece, Ireland, Italian (mainland + Sardinia), Moldova, Norway, Poland, Romania, Slovakia, Spain, Sweden, Switzerland, The Netherlands (doubtful), Ukraine; INPN: no data; additional data from Psyl'list: Algeria, China, Hungary, Japan, Lithuania, Mongolia, Russia, Slovenia.

\section{COMMENTS}

Description in Hodkinson \& White (1979a), Ossiannilsson (1992) and Burckhardt (2010). Biology in Conci et al. (1993). Host-plants: several Crataegus species, Malus domestica Borkh., Mespilus germanica L. and Pyrus communis L. (Rosaceae).

\section{Cacopsylla myrthi (Puton, 1876)}

Psylla myrthi Puton, 1876: 285.

Material eXAmined. - France. Alpes-Maritimes, Saint-MartinVésubie, M09-BOR1400-T1-M2, 1 9 (MNHN); Alpes-Maritimes, Saorge, M10-CAI1400-T1-M2, 1 으 (MNHN); same locality, M10CAI1400-T3-M2, $10^{\text {" }}, 1$ 우 (MNHN); same locality, M10-CAI1400-T4M2, 1 ㅇ (MNHN); same locality, M10-CAI1400-T6-M2, 2 우 (MNHN); same locality, M10-CAI1400-T3-M1, 1 ㅇ (MNHN); Alpes-Maritimes, Saorge, M10-CAI2000-T1-M1, 40", 1 우 (MNHN); same locality, M10-CAI2000-T3-M1, $50^{7}$, 4 우 (MNHN); same locality, M10-CAI2000-T4-M1, 30", 3 ㅇ (MNHN); same locality, M10-CAI2000-T5-M1, 407, 3 9 (MNHN); same locality, M10CAI2000-T1-M2, $10^{\prime \prime}, 1$ ㅇ (MNHN); same locality, M10-CAI2000-T2M2, $100^{\circ}, 6$ ( $\mathrm{PNHN}$ ); same locality, M10-CAI2000-T3-M2, $20^{\circ}$, 4 (BMNH); same locality, M10-CAI2000-T4-M2, $10^{7}$ (MNHN); same locality, M10-CAI2000-T5-M2, $10^{7}$ (MNHN); same locality,

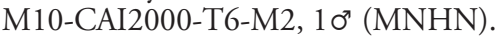

Distribution. - Fauna Europaea: France, Germany, Greece, Italy (mainland + Sicily), Malta, Spain, Ukraine; INPN: no data; additional data from Psyl'list: Algeria, Israel, Turkey.

\section{COMMENTS}

Description in Vondráček (1950) (as Psylla myrti Put.) and Loginova (1975) (as Psylla euxina Loginova, 1975). Biology in Conci et al. (1993). Host-plants: Rhamnus alaternus L. and Rhamnus palaestina Boiss. (Rhamnaceae).

\section{Cacopsylla notata (Flor, 1861)}

Psylla notata Flor, 1861: 365.

Material eXamined. - France. Alpes-Maritimes, Saint-Dalmasle-Selvage, M09-SES2000-T1-IN, 1 우 (MNHN); Alpes-Maritimes, Moulinet, Col de Turini, piste de Gasta-Fume, $1625 \mathrm{~m}$, $43^{\circ} 58^{\prime} 27.1482^{\prime \prime N}-7^{\circ} 23^{\prime} 42.7374$ "E, 02.VII.2008, C. Cocquem- 
pot (CBGP), 1 adult; Alpes-Maritimes, Moulinet, Col de Turini, $1610 \mathrm{~m}, 43^{\circ} 58^{\prime} 37.56^{\prime \prime} \mathrm{N}-7^{\circ} 23^{\prime} 30.7782^{\prime \prime E}$, 09.VII.2009, on herbaceous plants, C. Cocquempot (CBGP), 1 adult; Alpes-Maritimes, Saint-Dalmas-de-Tende, Refuge Neige et Merveilles, $1545 \mathrm{~m}$, 444'12.0318”N-7³0'44.064”E, 16.VII.2009, C. Cocquempot (CBGP), 2 adults; Alpes-de-Haute-Provence, Entraunes, Piste de la Cascade de l'Aiglière, 1420 m, 44¹2'24.9732"N-644'33.4782"E, 14.VII.2011, C. Cocquempot (CBGP), 1 adult; Alpes-Maritimes, Saint-Dalmas-de-Tende, Lac de Trem, 2270 m, 44³’5.0472”N7²6’36.8622”'E, 18.VII.2009, C. Cocquempot (CBGP), 1 adult.

DisTRIBUTION. - Fauna Europaea: Bulgaria, France, Greece, Italy (mainland + Sardinia + Sicily); INPN: no data; additional data from Psyl'list: Iran, Turkey.

\section{COMments}

Description in Burckhardt \& Hodkinson (1986). Biology in Conci et al. (1993). Host-plants: several species of Pyrus (Rosaeae).

\section{Cacopsylla picta (Foerster, 1848)}

Psylla picta Foerster, 1848: 81.

Material examined. - France. Alpes-Maritimes, Saorge, M10CAI1400-T5-M2, $10^{\circ}$ (MNHN).

Distribution. - Fauna Europaea: Austria, Belgium, Czech Republic, Finland, France, Germany, Italy, Moldova, Slovakia, Sweden, Switzerland, Ukraine; INPN: no data; additional data from Psyl'list: Bulgaria, Great Britain, Slovenia.

\section{COMMENTS}

Description in Ossiannilsson (1992), as Cacopsylla (Thamnopsylla) costalis (Flor, 1861), and Burckhardt (2010). Biology in Ossiannilsson (1992). Host-plants: several species of Malus (Rosaceae).

\section{Cacopsylla propinqua (Schaefer, 1949)} (Fig. 1A)

Psylla propinqua Schaefer, 1949: 37.

Material EXAmined. - France. Alpes-Maritimes, Saint-Dalmasde-Tende, Lac de la Muta, 2365 m, 442'57.2742”N-7²6'5.694”E, 18.VII.2009, C. Cocquempot (CBGP), 1 adult.

Distribution. - Fauna Europaea: Finland, Germany, Italy, Norway, Russia, Slovakia (doubtful), Sweden, Switzerland; INPN: no data; additional data from Psyl'list: no additional data.

\section{COMMENTS}

Description in Schaefer (1949) and Conci \& Tamanini (1986a). Biology in Conci et al. (1993). Host-plants: several species of Salix (Salicaceae). New for France.

\section{Cacopsylla pruni (Scopoli, 1763)}

Chermes pruni Scopoli, 1763: 140.
Material eXamined. - France. Alpes-Maritimes, Saorge, M10CAI1400-T1-M2, $10^{7}$ (MNHN); same locality, M10-CAI1400-T2M2, $10^{\prime \prime}, 1$ ㅇ (BMNH); same locality, M10-CAI1400-T6-M2, 1 \% (MNHN); same locality, M10-CAI2000-T1-M1, $10^{7}$ (MNHN); same locality, M10-CAI2000-T1-M2, $10^{7}$ (MNHN); AlpesMaritimes, Saint-Martin-Vésubie, M09-BOR1400-T1-M1, 1 ㅇ (MNHN).

Distribution. - Fauna Europaea: Austria, Belgium, Bulgaria, Czech Republic, Denmark, Finland, France, Germany, Great Britain, Ireland, Italy, Moldova, Norway, Poland, Romania, Russia, Slovakia, Spain, Sweden, Switzerland, Ukraine; INPN: no data; additional data from Psyl'list: Caucasus, Georgia, Hungary, Iran, Slovenia

\section{COMMENTS}

One specimen is noteworthy in lacking the typical orangebrown forewing coloration. Description in Hodkinson \& White (1979a), Ossiannilsson (1992) and Burckhardt (2010). Biology in Lauterer (1999; 2011). Host-plants: several Prunus species. This species is a vector of "Candidatus Phytoplasma prunorum responsible for the European Stone Fruit Yellows (Carraro et al. 1998). According to Peccoud et al. (2013), C. pruni is a complex of two cryptic species.

\section{Cacopsylla pulchella (Löw, 1877)}

Psylla pulchella Löw, 1877: 143.

Material eXamined. - France. Alpes-Maritimes, Saint-Martin-Vésubie, M09-BOR1400-T2-M2, $10^{\prime \prime}, 1$ \% (BMNH); AlpesMaritimes, Saint-Dalmas-le-Selvage, M09-SES1400-T8-M2, 1 \% (MNHN); M09-SES2000-T2-M2, $10^{\prime \prime}$ (MNHN); same locality, M09-SES2000-T8-M2, 1 ㅇ (MNHN).

Distribution. - Fauna Europaea: France, Germany, Great Britain, Greece, Italy (mainland + Sicily), Switzerland, Ukraine; INPN: no data; additional data from Psyl'list: Hungary, Israel, Lebanon, Portugal (mainland + Azores), Serbia, Slovenia, Spain (mainland + Balearic Islands), Turkey.

\section{COMMENTS}

Description in Hodkinson \& White (1979a) and Burckhardt (1999). Biology in Burckhardt (1999). Host-plant: Cercis siliquastrum L. (Fabaceae).

\section{Cacopsylla pulchra (Zetterstedt, 1840)}

Chermes pulchra Zetterstedt, 1840: 309.

Material eXAmined. - France. Alpes-Maritimes, Saint-MartinVésubie, M09-BOR1400-T2-M2, $10^{7}$ (MNHN).

DistribUtiON. - Fauna Europaea: Austria, Belgium, Czech Republic, Denmark, Finland, France, Germany, Great Britain, Ireland, Italy (mainland + Sardinia + Sicily), Moldova, Norway, Poland, Romania, Russia, Slovakia, Sweden, Switzerland, Ukraine; INPN: no data; additional data from Psyl'list: Caucasus, Hungary, Japan, Slovenia.

\section{COMMENTS}

Description in Hodkinson \& White (1979a) and Ossiannilsson (1992). Biology in Conci et al. (1993), Ossiannilsson 
(1992) and Lauterer (1998). Host-plants: several species of Salix (Salicaceae).

\section{Cacopsylla rhamnicola (Scott, 1876)}

Psylla rhamnicola Scott, 1876: 548.

Material EXAMINED. - France. Alpes-Maritimes, Saint-Dalmas-leSelvage, M09-SES1400-T7-M2, $10^{7}$ (MNHN); Alpes-Maritimes, Saorge, M10-CAI1400-T4-M2, 19 (MNHN).

Distribution. - Fauna Europaea: Austria, Czech Republic, Denmark, Finland, France, Great Britain, Ireland, Italy (mainland), Moldova, Russia, Slovakia, Slovenia, Sweden, Switzerland, Ukraine; INPN: no data; additional data from Psyl'list: Caucasus, Georgia, Hungary, Kazakhstan, Mongolia, Romania, Slovenia, Spain, Turkey.

\section{COMMENTS}

Description in Hodkinson \& White (1979a), Conci \& Tamanini (1988) and Ossiannilsson (1992). Biology in Ossiannilsson (1992) and Lauterer (1999). Host-plants: several species of Rhamnus (Rhamnaceae).

\section{Cacopsylla rhododendri (Puton, 1871)}

(Fig. 1B)

\section{Psylla rhododendri Puton, 1871: 436.}

Material eXAmined. - France. Alpes-Maritimes, Valdeblore, M09-BOR2000-T7-M1, 1 (MNHN); same locality, M09BOR2000-T8-M2, $30^{7}$ (MNHN); France. Alpes-Maritimes, Saorge, M10-CAI1400-T7-M2, 207 (BMNH); Alpes-Maritimes, Tende, Val de Minière, 1600 m, 10.VII.1994, 307, 19 ' I. Malenovský (MMBC); Alpes-Maritimes, Bolène-Vesubie, massif de la cime du Diable, 1800-2600 m, 28.VIII.1995, 907, 69, I. Malenovský (MMBC); Alpes-Maritimes, La Brigue, French-Italian border between Mt Grai $(2013 \mathrm{~m})$ and Cime de Marte (2135 m), 21.VIII.1996, 20", 1ㅇ, I. Malenovský (MMBC).

Distribution. - Fauna Europaea: Austria, Belgium, Finland, France, Italy, Moldova, Romania, Switzerland, Ukraine; INPN: no data; additional data from Psyl'list: "Czechoslovakia", Germany, Japan, Korea, Russia.

\section{COMMENTS}

Description in Dobreanu \& Manolache (1962) and Ossiannilsson (1992). Biology in Ossiannilsson (1992) and Conci et al. (1993). Host-plants: several species of Rhododendron (Ericaceae).

\section{Cacopsylla saliceti \\ (Foerster, 1848)}

Psylla saliceti Foerster, 1848: 79.

Material eXAmined. - France. Alpes-Maritimes, Valdeblore, M09-BOR2000-T8-M2, $10^{7}$ (MNHN); Alpes-Maritimes, SaintDalmas-le-Selvage, M09-SES2000-T1-M2, $10^{7}$ (BMNH); Alpes-
Maritimes, Breil-sur-Roya, Authion, vacherie de l'Ortiguier, $1960 \mathrm{~m}$, $43^{\circ} 59^{\prime} 40.6284$ "N-7²6'1.0536" E, on Larix decidua, 20.VII.2009, C. Cocquempot (CBGP), 7 adults; Alpes-Maritimes, Lantosque,

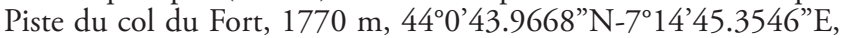
13.VII.2009, on Larix decidua Mill., C. Cocquempot (CBGP), 8 adults; Alpes-Maritimes, Lantosque, Col d'Andrion (Col du Fort), 1990 m, 441'3.8202"N-7¹5'12.1206”E, 13.VII.2009, on Larix decidua Mill., C. Cocquempot (CBGP), 1 adult; AlpesMaritimes, Saint-Dalmas-de-Tende, Pas de La Nauque, 2130 m,

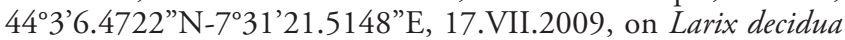
Mill., C. Cocquempot (CBGP), 2 adults.

Cacopsylla saliceti (Foerster, 1848)? Alpes-Maritimes, Valdeblore, Col de Salèse, coniferous forest, $2058 \mathrm{~m}, 44^{\circ} 8^{\prime} 14.424^{\prime \prime} \mathrm{N}-7^{\circ} 14^{\prime} 13.1274$ " E, 10.VI.09-24.VI.09 (Malaise trap, M09-BOR2000-T1-M1), 1 우 (MNHN).

Distribution. - Fauna Europaea: Austria, Belgium, Bulgaria, Czech Republic, France, Germany, Great Britain, Ireland, Italy (mainland + Sicily), Moldova, Poland, Romania, Slovakia, Spain, Switzerland, The Netherlands, Ukraine; INPN: no data; additional data from Psyl'list: Georgia, Hungary, Iran, Slovenia, Turkey.

\section{COMMENTS}

Description in Hodkinson \& White (1979a). Biology in Conci et al. (1993) and Lauterer (1999). Host-plants: several species of Salix (Salicaceae).

\section{Cacopsylla sorbi (Linnaeus, 1767)}

Chermes sorbi Linnaeus, 1767: 738.

Material examined. - France. Alpes-Maritimes, Moulinet,

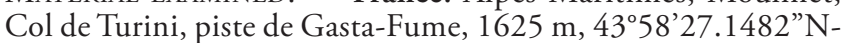
7²3’42.7374"E, 04.VII.2008, C. Cocquempot (CBGP), 1 adult; same data except 02.VII.2008, C. Cocquempot (CBGP), 127 adults; Alpes-Maritimes, Moulinet, Col de Turini, $1610 \mathrm{~m}$, 4358'37.56”N- 7²3'30.7782”E, 09.VII.2009, on herbaceous plants, C. Cocquempot (CBGP), 40 adults; Alpes-Maritimes, Lantosque, Col d'Andrion (Col du Fort), 1990 m, 44¹'3.8202"N7¹5'12.1206”'E, 13.VII.2009, on Sorbus aucuparia L., C. Cocquempot (CBGP), 41 adults; same data except on Larix decidua Mill., C. Cocquempot (CBGP), 2 adults; Alpes-Maritimes, Saint-Dalmas-de-Tende, GR du refuge des Merveilles, $1800 \mathrm{~m}$,

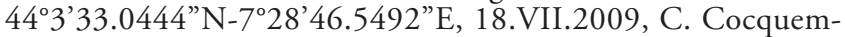
pot (CBGP), 35 adults; same data except Lac de la Minière, 1565 m, 444'9.9546"N-7³0'37.641"E, 17.VII.2009, on Salix sp., C. Cocquempot (CBGP), 7 adults; same data except GR du Pas de la Nauque, 1536 m, 444'6.7476”N-7³0'50.6232”E, 17.VII.2009, on Larix decidua Mill., C. Cocquempot (CBGP), 136 adults; Alpes-Maritimes, Saint-Dalmas-de-Tende, Pas de La Nauque, 2130 m, 44³'6.4722"N-7³1'21.5148”E, 17.VII.2009, on Larix decidua Mill., C. Cocquempot (CBGP), 2 adults; Alpes-Maritimes, Saint-Dalmas-de-Tende, Lac de Trem, $2270 \mathrm{~m}$, 443'5.0472"N-7²6'36.8622"E, 18.VII.2009, C. Cocquempot (CBGP), 4 adults; Breil-sur-Roya, Authion, vacherie de l'Ortiguier, 1960 m, 4359'40.6284"N-7²6'1.0536”'E, 20.VII.2009, on Larix decidua Mill., C. Cocquempot (CBGP), 4 adults; Alpesde-Haute-Provence, Colmars-les-Alpes, Piste de Ratéry, $1670 \mathrm{~m}$, $44^{\circ} 11$ '16.5804"N-639'15.4548”E, 10.VII.2011, on Sorbus aucuparia L., C. Cocquempot (CBGP), 14 adults; Alpes-Maritimes, Tende, Val de Minière, 1600 m, 10.VII.1994, $30^{7}, 10$ 옹 I. Malenovský (MMBC); Alpes-Maritimes, Bolène-Vesubie, massif de la cime du Diable, 1800-2600 m, 28.VIII.1995, $30^{\circ}$, 1 , I. Malenovský (MMBC). 
A
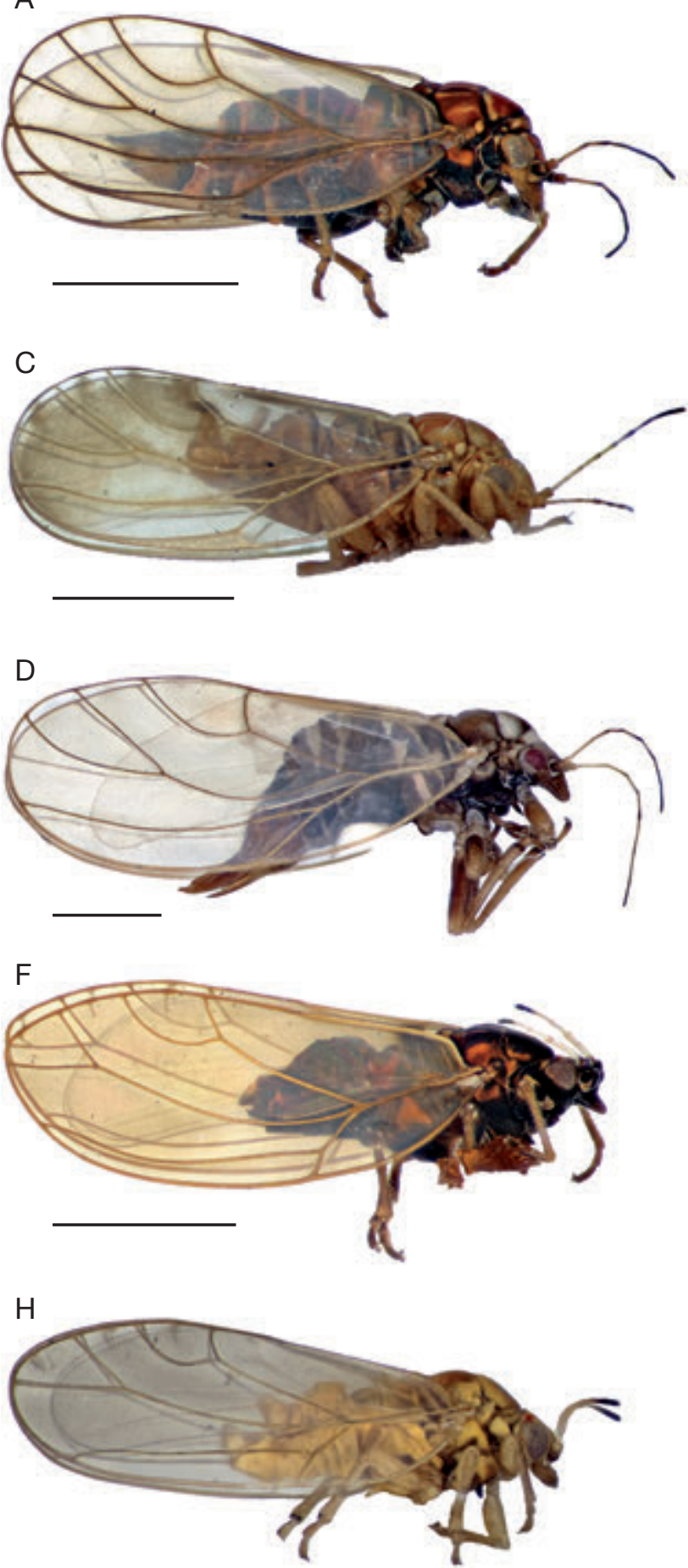

$\mathrm{B}$

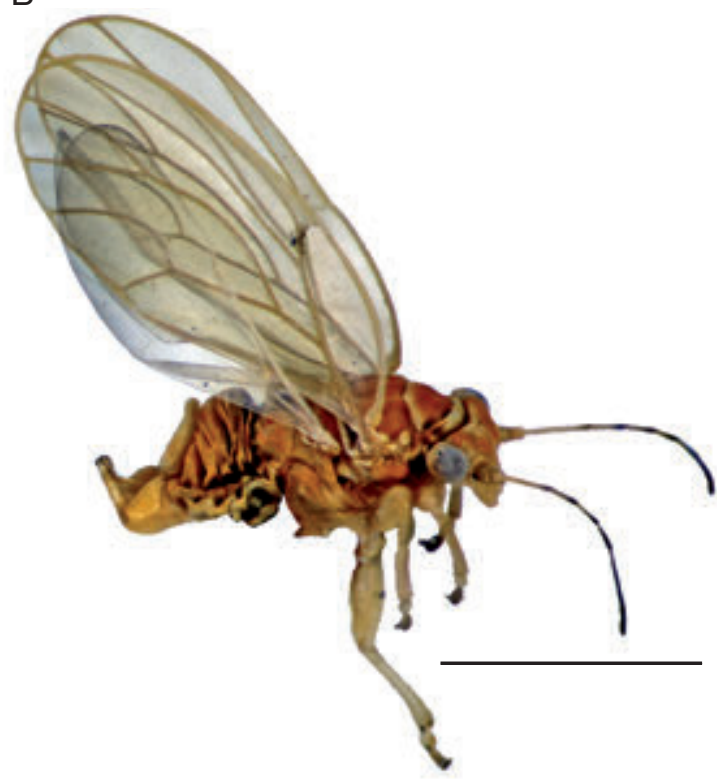

E
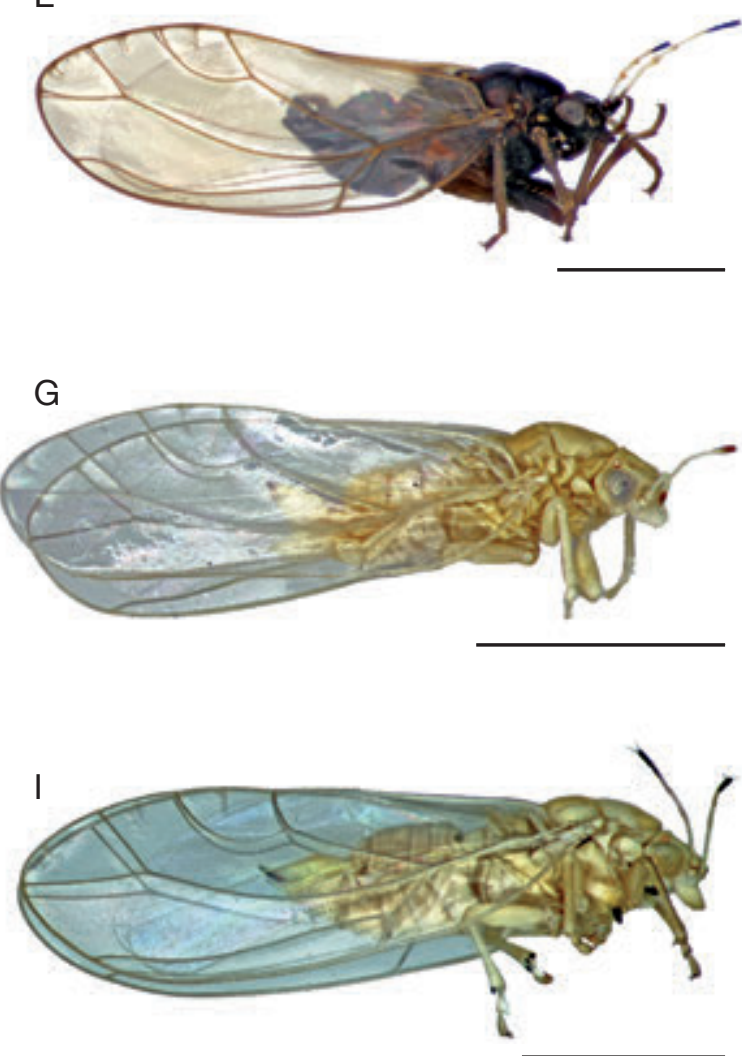

FIG 1. - Some noteworthy psyllid species collected in the Mercantour National Park. Species preceded by an asterisk are new for France. A, ${ }^{\star}$ Cacopsylla propinqua (Schaefer, 1949); B, Cacopsylla rhododendri (Puton, 1871); C, *Cyamophila prohaskai (Priesner, 1927); D, Psylla fusca (Zetterstedt, 1828); E, Bactericera bohemica (Šulc, 1913); F, Bactericera femoralis (Foerster, 1848); G, Trioza brachyceraea Hodkinson \& White, $1979=$ Trioza (Trioza) rapisardai Conci \& Tamanini, 1984 syn. nov.; H, Trioza cerastii (Linnaeus, 1758); I, *Trioza flixiana Burckhardt \& Lauterer, 2002. Scale bars: 1 mm.

Distribution. - Fauna Europaea: Austria, Belgium, Bulgaria, Czech Republic, Denmark, Finland, France, Germany, Great Britain, Ireland, Italy, Norway, Poland, Russia, Slovakia, Sweden, Switzerland, Ukraine; INPN: no data; additional data from Psyl'list: North America, Slovenia.

\section{COMments}

Description in Hodkinson \& White (1979a) and Ossiannilsson (1992). Biology in Ossiannilsson (1992), Conci et al. (1993) and Lauterer (1999). Host-plants: Sorbus aucuparia L. and Sorbus amurensis Koehne (Rosaceae). 


\section{Cacopsylla visci (Curtis, 1835)}

Psylla visci Curtis, 1835: unpaginated, with plate 565.

Material eXamined. - France. Alpes-Maritimes, Saint-MartinVésubie, M09-BOR1400-T1-M2, 19 (MNHN); same locality, M09-BOR1400-T8-M2, $10^{7}$ (MNHN); Alpes-Maritimes, Saorge, M10-CAI1400-T1-M1, $10^{\text {" }}$ (BMNH); same locality, M10CAI1400-T3-M2, 1 ( $(\mathrm{BMNH})$.

Distribution. - Fauna Europaea: Austria, Belgium, Czech Republic, France, Germany, Great Britain, Italy, Moldova, Poland, Slovakia, Switzerland, Ukraine; INPN: no data; additional data from Psyl'list: Bulgaria, Caucasus, Hungary, Iraq, Japan, Morocco, Slovenia.

\section{COMMENTS}

Description in Hodkinson \& White (1979a). Biology in Conci et al. (1993) and Lauterer (1999). Host-plants: Viscum album L. (Santalaceae) and Loranthus europaeus Jacq. (Loranthaceae).

\section{Cacopsylla zetterstedti (Thomson, 1877)}

Chermes zetterstedti Thomson, 1877: 832.

Material eXamined. - France. Alpes-de-Haute-Provence, Fours, Bayasse, 1790 m, 44¹8’39.366”N-644’23.0022”E, 11.VII.2011, on Hippophae rhamnoides L. [=Elaeagnus rhamnoides (L.) A. Nelson], C. Cocquempot (CBGP), 53 adults.

Distribution. - Fauna Europaea: Austria, Belgium, Czech Republic, Denmark, Finland, France, Germany, Great Britain, Italy, Norway, Poland, Slovakia, Sweden, Switzerland, The Netherlands; INPN: no data; additional data from Psyl'list: Caucasus, Georgia, Russia.

\section{COMMENTS}

Description in Hodkinson \& White (1979a) and Ossiannilsson (1992). Biology in Conci et al. (1993) and Lauterer (1999). Host-plant: Elaeagnus rhamnoides (L.) A. Nelson.

Genus Cyamophila Loginova, 1976

Cyamophila prohaskai (Priesner, 1927)

(Fig. 1C)

Psylla prohaskai Priesner, 1927: 263.

Material eXAmined. - France. Alpes-Maritimes, Saint-Dalmasle-Selvage, M09-SES2000-T8-M2, $10^{7}$ (MNHN).

Distribution. - Fauna Europaea: Austria, Italy, Switzerland; INPN: no data; additional data from Psyl'list: no additional data.

\section{COMMENTS}

Description in Conci \& Tamanini (1986b), Conci \& Tamanini (1989a) and Hodkinson \& Hollis (1987). Biology in Conci \& Tamanini (1989a) and Conci et al. (1993). Host-plant: Anthyllis vulneraria L. (Fabaceae). New for France.
Genus Livilla Curtis, 1835

Livilla pyrenaea (Mink, 1859)

Arytaina pyrenaea Mink, 1859: 430.

Material eXamined. - France. Alpes-Maritimes, Saorge, M10CAI1400-T1-M1, 80', 7\% (MNHN); samelocality, M10-CAI1400-T5M1, $10^{7}, 4$ ㅇ (MNHN); same locality, M10-CAI1400-T1-M2, 40", 10 ㅇ (MNHN); same locality, M10-CAI1400-T4-M2, 1 ( $(\mathrm{MNHN})$; same locality, M10-CAI2000-T1-M1, $110^{\prime \prime}, 8$ 은 (NHMB); same locality, M10-CAI2000-T3-M1, 1 우 (MNHN); same locality, M10-CAI2000-T4-M1, $10^{7}, 3$ 은 locality, M10-CAI2000-T5-M1, $10^{7}, 1$ 우 (MNHN); same locality, M10-CAI2000-T6-M1, 50", 2 ㅇ (BMNH); same locality, M10CAI2000-T1-M2), 290", 10 \% (MNHN), $20^{\prime \prime}, 2$ ㅇ (NHMB); same locality, M10-CAI2000-T4-M2, $10^{7}, 1$ (MNHN); same locality, M10-CAI2000-T5-M2, 207, 1 우 (MNHN); M10-CAI2000-T6M2, $10^{7}, 2$ \% (MNHN); Alpes-Maritimes, Saorge, Route du Caïros, $604 \mathrm{~m}, 43^{\circ} 59^{\prime} 49.1064$ "N- 7³0'22.9602” E, 19.VII.2009, on Fabaceae, C. Cocquempot (CBGP), 44 adults; Alpes-de-HauteProvence, Colmars-les-Alpes, Chemin de la forêt Saint-Jean, $1310 \mathrm{~m}$, $44^{\circ} 10^{\prime} 45.6852^{\prime \prime}$ N-6 $37^{\prime} 56.7078^{\prime \prime} \mathrm{E}, 13 . \mathrm{VII} .2011$, on Fabaceae, C. Cocquempot (CBGP), 17 adults; Alpes-de-Haute-Provence, Entraunes, Piste de la Cascade de l'Aiglière, $1420 \mathrm{~m}, 44^{\circ} 12^{\prime} 24.9732^{\prime} \mathrm{N}$ 644'33.4782”E, 14.VII.2011, on Fabaceae, C. Cocquempot (CBGP), 9 adults.

DisTRIBUTION. - Fauna Europaea: France, Italy, Spain; INPN: no data; additional data from Psyl'list: Algeria.

\section{COMMENTS}

Description in Hodkinson \& White (1979a) and Hodkinson \& Hollis (1987). Biology in Conci \& Tamanini (1988) and Conci et al. (1993). Host-plants: several species of Genista (Fabaceae).

\section{Livilla variegata (Löw, 1881)}

Floria variegata Löw, 1881: 261.

Material eXamined. - France. Alpes-Maritimes, Saint-Dalmas-le-Selvage, M09-SES1400-T1-IN, $10^{7}, 1$ 으 (MNHN); same locality, M09-SES1400-T8-IN, 1 \% (MNHN); same locality, M09SES1400-T7-M1, 4 O (BMNH); same locality, M09-SES1400-T1M2, 1 ( (MNHN); Alpes-Maritimes, Saorge, M10-CAI1400-T4-M1, 1 ( $(\mathrm{MNHN})$; Alpes-Maritimes, Lantosque, Granges de la Brasque,

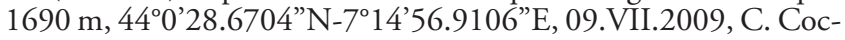
quempot (CBGP), 25 adults; Alpes-Maritimes, Moulinet, Col de Turini, piste de Gasta-Fume, $1625 \mathrm{~m}, 43^{\circ} 58^{\prime} 27.1482^{\prime} \mathrm{N}$ 7²3’42.7374"E, 02.VII.2008, C. Cocquempot (CBGP), 3 adults; Alpes-Maritimes, Moulinet, Col de Turini, 1610 m, 4358'37.56”N7²3'30.7782"E, 09.VII.2009, on herbaceous plants, C. Cocquempot (CBGP), 1 adult; Alpes-Maritimes, Saint-Dalmas-de-Tende, GR du Pas de la Nauque, 1536 m, 444'6.7476”N-7³0'50.6232”E, 17.VII.2009, on Fabaceae, C. Cocquempot (CBGP), 8 adults; Alpes-Maritimes, Tende, Les Mesches, 1450 m, 10.VII.1994, 1 운, I. Malenovský (MMBC).

Distribution. - Fauna Europaea: Austria, Czech Republic, France, Germany, Great Britain, Italy, Romania, Slovenia, Spain, Switzerland; INPN: no data; additional data from Psyl'list: Bosnia-Herzegovina, Canada (Newfoundland), Hungary, Slovakia. 


\section{COMMENTS}

Description in Hodkinson \& Hollis (1980) and Hodkinson \& Hollis (1987). Biology in Conci et al. (1993) and Malenovský \& Kment (2004). Host-plants: Laburnum alpinum (Mill.) Bercht. \& J. Presl and Laburnum anagyroides Medik. (Fabaceae).

Genus Psylla Geoffroy, 1762

Psylla alni (Linnaeus, 1758)

Chermes alni Linnaeus, 1758: 454.

Material examined. - France. Alpes-de-Haute-Provence, Enchastrayes, La Rente, 1702 m, 4421'14.022"N- 641'56.778”E, 29.VIII.2012, on Alnus sp., J.-C. Streito (CBGP), 8 adults; Alpes-de-

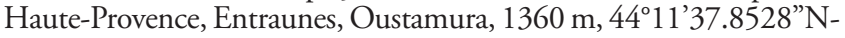
644'27.6144”'E, 14.VII.2011, on Alnus sp., C. Cocquempot (CBGP), 1 adult; Alpes-Maritimes, Lantosque, Saint-Colomban, Camari,

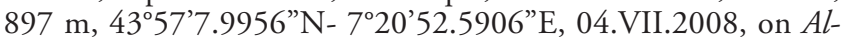
nus sp., C. Cocquempot (CBGP), 3 adults; Alpes-de-Haute-Provence, Entraunes, Le Clôt, 1324 m, 44¹1'47.0724”N-644'40.9878”E, 11.VII.2011, C. Cocquempot (CBGP), 18 adults; Alpes-de-HauteProvence, Fours, Tourbière des Girards, $1800 \mathrm{~m}, 4^{\circ} 18^{\prime} 41.8314^{\prime} \mathrm{N}-$ 644’7.3566”E, 01.IX.2012, J.-C. Streito (CBGP), 3 adults.

Distribution. - Fauna Europaea: Austria, Belgium, Bulgaria, Czech Republic, Denmark, Finland, France, Germany, Great Britain, Ireland, Italy (mainland + Sardinia), Moldova, Norway, Poland, Romania, Russia, Slovakia, Spain, Sweden, Switzerland, The Netherlands, Ukraine; INPN: no data; additional data from Psyl'list: Canada (Alberta, British Columbia), China, Greenland, Hungary, Japan, Korea, Slovenia, Taiwan, United States of America (Arizona, California, Idaho, Nevada, Oregon, Washington).

\section{COMMENTS}

Description in Hodkinson \& White (1979a) and Ossiannilsson (1992). Biology in Ossiannilsson (1992), Conci et al. (1993) and Lauterer $(1976,1998)$. Host-plants: several species of Alnus (Betulaceae).

\section{Psylla alpina Foerster, 1848}

Psylla alpina Foerster, 1848: 81.

Material examined. - France. Alpes-Maritimes, La Brigue, French-Italian border between Mt Grai $(2013 \mathrm{~m})$ and Cime de Marte (2135 m), 21.VIII.1996, 150", 6요, I. Malenovský (MMBC).

Distribution. - Fauna Europaea: Austria, Czech Republic, France, Germany, Italy, Poland, Romania, Switzerland, Ukraine; INPN: no data; additional data from Psyl'list: Bulgaria, Slovenia.

\section{COMMENTS}

Description in Dobreanu \& Manolache (1962). Biology in Lauterer (1979) and Conci et al. (1993). Host-plant: Alnus viridis (Chaix) DC. (Betulaceae).

Psylla buxi (Linnaeus, 1758)

Chermes buxi Linnaeus, 1758: 454.
Material examined. - France. Alpes-Maritimes, Valdeblore, M09-BOR2000-T2-M1, $10^{7}$ (MNHN); Alpes-Maritimes, SaintDalmas-le-Selvage, M09-SES2000-T2-M1, $10^{\circ}$ (BMNH); same locality, M09-SES2000-T2-M2, 30", 1 우 (BMNH); same locality, M09-SES1400-T1-M2, 1 ㅇ (MNHN); Alpes-Maritimes, Saorge, Forêt de Caïros, M10-CAI1400-T1-M2, 1 ㅇ (MNHN); same locality, M10CAI2000-T1-M1, $20^{7}$ (MNHN); same locality, M10-CAI2000-T3M1, 1 으 (MNHN); same locality, M10-CAI2000-T1-M2, $10^{7}, 2$ 우 (MNHN); Alpes-de-Haute-Provence, Entraunes, Le Clôt, 1324 m,

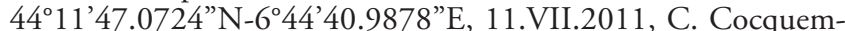
pot (CBGP), 1 adult; Alpes-de-Haute-Provence, Fours, Col de la Petite-Cayolle, $2400 \mathrm{~m}, 4^{\circ} 15^{\prime} 32.9286^{\prime \prime N}-6^{\circ} 44$ '29.709"E, 09.VII.2011, C. Cocquempot (CBGP), 6 adults; Alpes-de-HauteProvence, Entraunes, Piste de la Cascade de l'Aiglière, $1420 \mathrm{~m}$, $44^{\circ} 12^{\prime 2} 4.9732^{\prime \prime N}-6^{\circ} 44^{\prime} 33.4782^{\prime \prime E}$, 14.VII.2011, on Buxus sp., C. Cocquempot (CBGP), 9 adults; Alpes-Maritimes, Saorge, Route du Caïros, 604 m, 4359'49.1064”N- 7³0'22.9602”'E, 20.VII.2009, on Buxus sp., C. Cocquempot (CBGP), 3 adults.

Distribution. - Fauna Europaea: Austria, Belgium, Bulgaria, Czech Republic, Denmark, France, Germany, Great Britain, Ireland, Italy, Moldova, Norway, Poland, Romania, Slovakia, Spain, Sweden, Switzerland, The Netherlands, Ukraine; INPN: no data; additional data from Psyl'list: Canada (Newfoundland), Hungary, Slovenia, United States of America (Hawaii).

\section{COMMENTS}

Description in Hodkinson \& White (1979a) and Ossiannilsson (1992). Biology in Ossiannilsson (1992), Conci et al. (1993) and Lauterer (1998). Host-plants: several species of Buxus (Buxaceae).

\section{Psylla fusca (Zetterstedt, 1828)}

(Fig. 1D)

Chermes fusca Zetterstedt, 1828: 552.

Material eXAmined. - France. Alpes-de-Haute-Provence, Entraunes, Le Clôt, 1324 m, 44¹1'47.0724"N-644'40.9878”E, 11.VII.2011, C. Cocquempot (CBGP), 3 adults.

Distribution. - Fauna Europaea: Austria, Czech Republic, Denmark, Finland, France, Germany, Italy, Moldova, Norway, Poland, Romania, Russia, Slovakia, Sweden, Switzerland, Ukraine; INPN: no data; additional data from Psyl'list: Georgia, Latvia, Slovenia.

\section{COMMENTS}

Description in Ossiannilsson (1992). Biology in Ossiannilsson (1992), Conci et al. (1993) and Lauterer (1998). Host-plants: Alnus incana (L.) Moench (Betulaceae).

\section{Genus Spanioneura Foerster, 1848}

Spanioneura fonscolombii Foerster, 1848

Spanioneura fonscolombii Foerster, 1848: 94.

Material eXamined. - France. Alpes-Maritimes, Saint-Martin-Vésubie, M09 BOR1400-T1-M2, $20^{7}$ (MNHN); same locality, M09-BOR1400-T2-M2, $10^{\text {" }}$ (MNHN); same locality, M09-BOR1400-T2-M1, $10^{7}$ (MNHN); Alpes-Maritimes, Valdeblore, M09-SES2000-T1-M1, 1 우 (MNHN); same locality, M09- 
SES2000-T2-M1, 1 (MNHN); same locality, M09-SES2000-T2-M2, $10^{7}$ (MNHN); Alpes-Maritimes, Saint-Dalmas-le-Selvage, M09SES2000-T1-M1, 1 \% (MNHN); same locality, M09-SES2000-T2M1, $40^{\circ}, 4$ ㅇ (MNHN); same locality, M09-SES2000-T2-M2, 30", 19 (MNHN); Alpes-Maritimes, Saorge, M10-CAI1400-T1-M1, 1 은 (MNHN); same locality, M10-CAI1400-T3-M1, $20^{\circ}, 1$ ㅇ (MNHN); same locality, M10-CAI1400-T1-M2, 20" (MNHN); same locality, M10-CAI1400-T3-M2, $10^{7}, 3 \%$ (MNHN); AlpesMaritimes, Saorge, M10-CAI2000-T1-M1, 1 ( $(\mathrm{MNHN})$; same locality, M10-CAI2000-T2-M1, 1 우 (MNHN); same locality, M10CAI2000-T3-M, $10^{\prime \prime}, 2$ ( (MNHN); same locality, M10-CAI2000-T5M1, $10^{7}$ (MNHN); same locality, M10-CAI2000-T1-M2, $20^{\circ}, 1$ 우 (BMNH); same locality, M10-CAI2000-T3-M2, 20" (MNHN); same locality, M10-CAI2000-T4-M2, 1 으 (MNHN); same locality, M10-CAI2000-T3-IN, 1 \% (MNHN); Breil-sur-Roya, Authion, vacherie de l'Ortiguier, $1960 \mathrm{~m}$, 4359'40.6284"N-7²6'1.0536" E, 20.VII.2009, on Larix decidua Mill., C. Cocquempot (CBGP), 1 adult; Alpes-Maritimes, Moulinet, Col de Turini, 1610 m, 435' $37.56^{\prime \prime} \mathrm{N}$ 7²3'30.7782"E, 09.VII.2009, on herbaceous plants, C. Cocquempot (CBGP), 3 adults; Alpes-Maritimes, Lantosque, Granges de la Brasque, $1690 \mathrm{~m}, 4^{\circ} 0^{\prime} 28.6704^{\prime \prime N}-7^{\circ} 14$ '56.9106”'E, 09.VII.2009, on Salix sp., C. Cocquempot (CBGP), 1 adult; Alpes-Maritimes, Lantosque, Col d'Andrion (Col du Fort), 1990 m, 44²'3.8202"N$7^{\circ} 15^{\prime} 12.1206$ ”E, 13.VII.2009, on Larix decidua Mill., C. Cocquempot (CBGP), 1 adult; Alpes-de-Haute-Provence, Entraunes, Piste de la Cascade de l'Aiglière, $1420 \mathrm{~m}, 44^{\circ} 12^{\prime} 24.9732^{\prime \prime N}-6^{\circ} 44^{\prime} 33.4782^{\prime} \mathrm{E}$, 14.VII.2011, on Buxus sp., C. Cocquempot (CBGP), 5 adults; Alpes-de-Haute-Provence, Fours, Col de la Petite-Cayolle, $2400 \mathrm{~m}$,

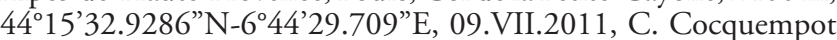
(CBGP), 1 adult; Alpes-Maritimes, Saint-Dalmas-de-Tende, Lac de la Muta, 2365 m, 44²'57.2742”N-7²6'5.694”E, 18.VII.2009, C. Cocquempot (CBGP), 6 adults; Alpes-Maritimes, Saorge, Route du Caïros, 604 m, 4359’49.1064”N- 7³0’22.9602”E, 20.VII.2009, on Buxus sp., C. Cocquempot (CBGP), 2 adults; Alpes-Maritimes, Saint-Dalmas-de-Tende, Lac de Trem, 2270 m, 44³'5.0472"N7²6’36.8622”E, 18.VII.2009, C. Cocquempot (CBGP), 9 adults.

Distribution. - Fauna Europaea: Belgium, France, Great Britain, Italy, Luxembourg, Spain, Switzerland; INPN: no data; additional data from Psyl'list: Ireland, Slovenia, United States of America (Connecticut).

\section{COMMENTS}

Description in Hodkinson \& White (1979a). Biology in Conci et al. (1993). Host-plant: Buxus sempervirens L. (Buxaceae).

Family TRIOZIDAE Löw, 1879

Genus Bactericera Puton, 1876

Bactericera bohemica (Šulc, 1913)

(Fig. 1E)

Trioza bohemica Šulc, 1913: 34.

Material eXAmined. - France. Alpes-Maritimes, Lantosque, Col d'Andrion (Col du Fort), 1990 m, 44¹'3.8202"N-7 15'12.1206”'E, 13.VII.2009, on Epilobium angustifolium L., C. Cocquempot (CBGP), 1 adult; Alpes-Maritimes, Lantosque, Granges de la Brasque, $1690 \mathrm{~m}, 44^{\circ} 0^{\prime} 28.6704^{\prime} \mathrm{N}-7^{\circ} 14 ' 56.9106 ” \mathrm{E}, 13 . \mathrm{VII} .2009$, C. Cocquempot (CBGP), 2 adults.

Distribution. - Fauna Europaea: Austria, Bulgaria, Czech Republic, Finland, France, Germany, Italy, Norway, Poland, Russia, Slovakia, Sweden, Switzerland, Ukraine; INPN: no data; additional data from Psyl'list: Canada, Caucasus, Estonia, Romania.

\section{COMMENTS}

Description in Šulc (1913), Conci \& Tamanini (1985) and Ossiannilsson (1992). Biology in Ossiannilsson (1992) and Conci et al. (1996). Host-plants: Geum montanum L. and $G$. rivale L. (Rosaceae).

\section{Bactericera curvatinervis}

(Foerster, 1848)

Trioza curvatinervis Foerster, 1848: 83.

Material eXamined. - France. Alpes-Maritimes, Saorge, M10CAI2000-T7-M1, 1 ㅇ (MNHN); Alpes-Maritimes, Moulinet, Col de Turini, piste de Gasta-Fume, $1625 \mathrm{~m}, 43^{\circ} 58^{\prime} 27.1482$ "N7²3’42.7374”'E, 02.VII.2008, C. Cocquempot (CBGP), 5 adults; Alpes-Maritimes, Lantosque, Col d'Andrion (Col du Fort), 1990 m, $44^{\circ} 1$ '3.8202"N- 7 $15^{\prime} 12.1206^{\prime \prime E}$, 13.VII.2009, on Epilobium angustifolium L., C. Cocquempot (CBGP), 1 adult.

Distribution. - Fauna Europaea: Austria, Belgium, Bulgaria, Czech Republic, Finland, France, Germany, Italy, Norway, Poland, Romania, Russia, Slovakia, Sweden, Switzerland; INPN: no data; additional data from Psyl'list: Caucasus, Georgia, Great Britain, Hungary, Ireland, Japan, Lebanon, Slovenia.

\section{COMMENTS}

Description in Hodkinson \& White (1979a) and Ossiannilsson (1992). Biology in Ossiannilsson (1992) and Conci et al. (1996). Host-plants: several species of Salix (Salicaceae).

\section{Bactericera femoralis (Foerster, 1848)} (Fig. 1F)

Trioza femoralis Foerster, 1848: 86.

Material EXAMINED, - France. Alpes-Maritimes, Saint-Dalmasle-Selvage, M09-SES2000-T1-IN, 1 \% (MNHN); Alpes-Maritimes, Saorge, Malaise trap, M10-CAI2000-T5-M1, $10^{7}$ (MNHN); same locality, M10-CAI2000-T6-M1, 1 으 (BMNH); same locality, M10CAI2000-T7-M1, $10^{7}$, 4 우 (MNHN), $10^{7}$ (BMNH); Alpes-Maritimes, Bolène-Vesubie, massif de la cime du Diable, 1800-2600 m, 28.VIII.1995, $30^{7}$, 4ㅇ, I. Malenovský (MMBC); Alpes-Maritimes, La Brigue, French-Italian border between Mt Grai (2013 m) and Cime

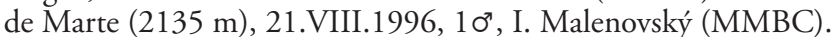

Distribution. - Fauna Europaea: Austria, Belgium, Czech Republic, Finland, France, Germany, Italy, Moldova, Norway, Poland, Romania, Russia, Slovakia, Sweden, Switzerland, The Netherlands, Ukraine; INPN: no data; additional data from Psyl'list: Bulgaria, Caucasus, Georgia, Slovenia, Turkey.

\section{COMMENTS}

Description in Ossiannilsson (1992). Biology in Bin (1972). Host-plants: several species of Alchemilla (Rosaceae).

\section{Bactericera parastriola}

Conci, Ossiannilsson \& Tamanini, 1988

Bactericera parastriola Conci, Ossiannilsson \& Tamanini, 1988: 226. 
Material eXamined. - France. Alpes-Maritimes, Saint-Dalmas-leSelvage, «Vallon de Restefond», alpine meadow, 2500 m, 16.VI.1994 (Malaise trap),, C. Cocquempot (CBGP), $10^{\prime \prime}$.

Distribution. - Fauna Europaea: Czech Republic, Italy, Romania, Sweden, Switzerland; INPN: no data; additional data from Psyl'list: Austria, Poland.

\section{COMMENTS}

Description in Conci et al. (1988) and Ossiannilsson (1992). Description of the fifth instar immature in Burckhardt \& Lauterer (1997). Biology in Conci et al. (1988), Ossiannilsson (1992) and Lauterer \& Malenovský (2002). Host-plants: several species of Salix (Salicaceae). New for France.

Genus Eryngiofaga Klimaszewski, 1968

Eryngiofaga cf. refuga (Loginova, 1966)

Trioza refuga Loginova, 1966: 28.

Material eXAmined. - France. Alpes-Maritimes, Saorge, M10CAI2000-T2-M2, $10^{\circ}(\mathrm{MNHN})$.

DisTRIBUTION. - Fauna Europaea: No data; INPN: no data; additional data from Psyl'list: Kazakhstan, Russia (West Siberia).

\section{COMMENTS}

Description in Loginova (1966). No data on biology (reported from the subalpine region of Kazakhstan: 2000-2500 m). Host-plant unknown. New for France. The single male at hand resembles Eryngiophaga refuga from Kazakhstan and Siberia. However, more material is required to decide if the French population is conspecific with those from Kazakhstan and Siberia.

Genus Trichochermes Kirkaldy, 1904

Trichochermes walkeri (Foerster, 1848)

Trioza walkeri Foerster, 1848: 88.

Material EXAMINED. - France. Alpes-Maritimes, Saint-Dalmasle-Selvage, M09-SES1400-T8-M2, 1 \% (MNHN).

Distribution. - Fauna Europaea: Austria, Belgium, Czech Republic, Denmark, France, Germany, Great Britain, Italy, Luxembourg, Moldova, Poland, Romania, Slovakia, Spain, Sweden, Switzerland, Ukraine; INPN: no data; additional data from Psyl'list: Estonia, Finland, Hungary, Latvia, Slovenia.

\section{COMMENTS}

Description in Hodkinson \& White (1979a) and Ossiannilsson (1992). Biology in Ossiannilsson (1992) and Conci et al. (1996). Host-plants: several species of Rhamnus (Rhamnaceae).
Genus Trioza Foerster, 1848

\section{Trioza abdominalis Flor, 1861}

Trioza abdominalis Flor, 1861: 502.

Material eXAmined. - France. Alpes-Maritimes, Saint-Dalmas-leSelvage, M09-SES1400-T7-M2, $10^{\prime \prime}, 1$ 을 (BMNH); Alpes-Maritimes, Saorge, M10-CAI1400-T1-M2, 1 ㅇ (MNHN); same locality, M10CAI2000-T7-M1, 40", 2 ㅇ (MNHN); Alpes-Maritimes, BolèneVesubie, massif de la cime du Diable, 1800-2600 m, 28.VIII.1995, $10^{*}, 1$ ㅇ, I. Malenovský (MMBC).

Distribution. — Fauna Europaea: Austria, Czech Republic, Finland, France, Germany, Great Britain, Italy (mainland + Sicily), Norway, Poland, Russia, Slovakia, Spanish (mainland + Canary Is.), Sweden, Switzerland; INPN: no data; additional data from Psyl'list: Estonia, Korea, Latvia, Mongolia, Slovenia.

\section{COMMENTS}

Description in Hodkinson \& White (1979a) and Ossiannilsson (1992). Biology in Ossiannilsson (1992) and Conci et al. (1996). Host-plants: several Achillea and Chrysanthemum spp. (Asteraceae).

Trioza anthrisci Burckhardt, 1986

Trioza anthrisci Burckhardt, 1986: 425.

Material eXAmined. - France. Alpes-Maritimes, Saint-MartinVésubie, M09-BOR1400-T2-M1, $20^{\star \prime}$ (MNHN).

Distribution. - Fauna Europaea: Austria, Czech Republic, Finland, France, Germany, Italy, Norway, Poland, Romania, Russia, Slovakia, Sweden, Switzerland; INPN: no data; additional data from Psyl'list: Slovenia, Turkey, Ukraine.

\section{COMMENTS}

Description in Burckhardt (1986). Biology in Conci et al. (1996). Host-plants: Anthriscus sylvestris (L.) Hoffm., Angelica sylvestris L., Chaerophyllum hirsutum subsp. villarsii (Koch) Brique and Heracleum sphondylium L. (Apiaceae).

\section{Trioza brachyceraea}

Hodkinson \& White, 1979

(Fig. 1G)

Trioza brachyceraea Hodkinson \& White, 1979: 61; holotype male, France: Lozère, Gorges du Tarn, 25.V.1977 - Genista hispanica (I. M. White), (BMNH, slide mounted, examined).

Trioza (Trioza) rapisardai Conci \& Tamanini, 1984: 201; holotype male, Italy: Piedmont, Susa Valley, near Cesana Torinese, 1330 1550 m, 1.VII.1984, Laserpitium siler (C. Conci) (Museo Civico di Storia Naturale di Milano, dry mounted, not examined). N. syn.

Material eXAmined. - France. Alpes-Maritimes, Saint-MartinVésubie, M09-BOR1400-T7-M1, 1 O (MNHN); Alpes-Maritimes, Saint-Dalmas-le-Selvage, M09-SES1400-T1-M2), $10^{\prime \prime}, 3$ 으 (MNHN); same locality, M09-SES1400-T8-M2, 1 (MNHN); Alpes-Maritimes, Saorge, M10-CAI2000-T7-M1, 1 (BMNH). 
DisTRIBUTION. - Fauna Europaea: France (report for T. brachycereae [sic]), Italy (report for T. rapisardai); INPN: no data; additional data from Psyl'list: no additional data.

\section{COMMENTS}

Description in Hodkinson \& White (1979b) and Conci \& Tamanini (1984). Biology in Conci \& Tamanini (1984) and Conci et al. (1996). Host-plant: Laserpitium siler L. and Laserpitium gallicum L. (Apiaceae). Trioza brachyceraea has been known only from the holotype from France, while T. rapisardai was known from three places in the Susa Valley, Piedmont, in northern Italy. A comparison of the holotype of $T$. brachyceraea with paratypes of T. rapisardai housed in the collections of the BMNH and MHNG showed that they are conspecific. We therefore consider T. rapisardai to be a junior subjective synonym of $T$. brachyceraea.

\section{Trioza caesaris}

Burckhardt \& Lauterer, 2002

Trioza caesaris Burckhardt \& Lauterer, 2002: 22.

MATERIAL EXAMINED. - France. Alpes-Maritimes, Saint-Dalmasle-Selvage, M09-SES2000-T8-M2, $10^{7}$ (MNHN).

Distribution. - Fauna Europaea: Austria, Czech Republic, France, Italy, Switzerland; INPN: observed in 1986 in Department of HautRhin (data CardObs; square 10 kmL93E100N677); additional data from Psyl'list: no additional data.

\section{COMMENTS}

Description and biology in Burckhardt \& Lauterer (2002a). Host-plant: Cardamine hirsuta L. (Brassicaceae).

\section{Trioza carpathica}

Dobreanu \& Manolache, 1959

Trioza apicalis carpathica Dobreanu \& Manolache, 1959: 295.

Material EXAMINED. — France. Alpes-Maritimes, Saint-Dalmasle-Selvage, M09-SES1400-T1-IN, 1 우 (MNHN).

Distribution. - Fauna Europaea: France, Romania; INPN: no data; additional data from Psyl'list: no additional data.

\section{COMMenTs}

Description in Dobreanu \& Manolache $(1959,1962)$ and Burckhardt (1986). No data on biology. Host-plants: Angelica archangelica L. and Daucus carota L. (Apiaceae).

\section{Trioza centranthi (Vallot, 1829)}

Psylla centranthi Vallot, 1829: 106.

Material eXamined. - France. Alpes-de-Haute-Provence, Entraunes, Piste de la Cascade de l'Aiglière, 1420 m, 44 ${ }^{\circ} 12^{\prime} 24.9732^{\prime \prime} \mathrm{N}$ 644'33.4782"E, 14.VII.2011, on Centranthus angustifolius (Mill.) DC., C. Cocquempot (CBGP), 72 adults.
Distribution. - Fauna Europaea: Austria, Belgium, Czech Republic, Denmark, France, Germany, Great Britain, Greece, Italy (mainland + Sardinia + Sicily), Malta, Moldova, Poland, Romania, Slovakia, Switzerland, Ukraine; INPN: observed in 2009 in Mercantour National Parc (data ATBI Mercantour; square 10 kmL93E106N633); additional data from Psyl'list: Algeria, Caucasus, Hungary, Morocco, Slovenia, ex-Yugoslavia.

\section{COMMENTS}

Description in Hodkinson \& White (1979a), Ossiannilsson (1992) and Burckhardt \& Lauterer (2006). Biology in Ossiannilsson (1992) and Conci et al. (1996). Host-plants: several species of Centranthus and Valerianella, as well as Fedia cornucopiae Gaertn. (Caprifoliaceae).

\section{Trioza cerastii (Linnaeus, 1758)}

(Fig. 1H)

Chermes cerastii Linnaeus, 1758: 454.

Material eXAMINED. - France. Alpes-Maritimes, Valdeblore, M09BOR2000-T7-M1, $10^{\top 7}$ (MNHN); Alpes-Maritimes, Saint-Dalmasle-Selvage, M09-SES2000-T7-M2, 1 ( Saorge, M10-CAI2000-T5-M1, 1 ( CAI2000-T6-M2, $10^{\prime \prime}, 1$ ( (BMNH); Alpes-Maritimes, Lantosque,

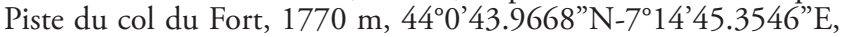
13.VII.2009, on Larix decidua Mill., C. Cocquempot (CBGP), 17 adults; Alpes-Maritimes, Lantosque, Granges de la Brasque, 1690 m, 440'28.6704”N-7¹4'56.9106”E, 13.VII.2009, C. Cocquempot (CBGP), 17 adults; Alpes-Maritimes, Bolène-Vesubie,

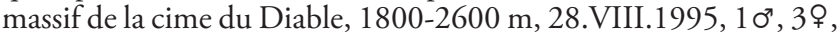
I. Malenovský (MMBC); Alpes-Maritimes, La Brigue, French-Italian border between Mt Grai (2013 m) and Cime de Marte (2135 m), 21.VIII.1996, 1요, I. Malenovský (MMBC).

Distribution. - Fauna Europaea: Austria, Czech Republic, Denmark, Finland, France, Germany, Italy, Norway, Poland, Romania, Russia, Slovakia, Spain (Balearic Is.), Sweden, Switzerland, Ukraine; INPN: no data; additional data from Psyl'list: Bulgaria, China, Estonia, Hungary, Slovenia, Spain.

\section{COMMENTS}

Description in Ossiannilsson (1992). Biology in Ossiannilsson (1992) and Conci et al. (1996). Host-plants: several species of Cerastium (Caryophyllaceae).

\section{Trioza cirsii Löw, 1881}

Trioza cirsii Löw, 1881: 264.

Material eXAmined. - France. Alpes-Maritimes, Saorge, M10CAI1400-T5-M1, $10^{\circ}$ (MNHN); same locality, M10-CAI1400-T6$\mathrm{M} 2,1$ ㅇ (MNHN).

Distribution. - Fauna Europaea: Austria, Bulgaria, Czech Republic, Denmark, Finland (doubtful), France, Germany, Greece, Moldova, Norway, Poland, Romania, Russia, Slovakia, Sweden, Switzerland, Ukraine; INPN: no data; additional data from Psyl'list: Slovenia.

\section{COMMENTS}

Description in Ossiannilsson (1992) and Burckhardt \& Lauterer (2002b). Biology in Ossiannilsson (1992) and Lauterer (1994). Host-plants: several species of Cirsium (Asteraceae). 
Trioza flixiana Burckhardt \& Lauterer, 2002 (Fig. 1I)

Trioza flixiana Burckhardt \& Lauterer, 2002: 800.

Material eXAmined. - France. Alpes-Maritimes, Saint-MartinVésubie, M09-BOR1400-T1-M1, 1 우 (MNHN); same locality, M09-BOR1400-T2-M1, 1 으 (BMNH).

Distribution. - Fauna Europaea: Austria, Czech Republic, Italy, Slovakia, Switzerland; INPN: no data; additional data from Psyl'list: Slovenia.

\section{COMMENTS}

Also reported from Spain on the GBIF portal. Description and elements of biology in Burckhardt \& Lauterer (2002b). Host-plant: probably Cirsium spinosissimum (L.) Scop. (Asteraceae). New for France.

\section{Trioza kiefferi Giard, 1902}

Trioza kiefferi Giard, 1902: 121.

MATERIAl EXAMINED. - France. Alpes-Maritimes, Saorge, Malaise trap, M10-CAI1400-T6-M2, 1 오 (MNHN).

DiSTRIBUTION. - Fauna Europaea: Italy (mainland + Sicily), Malta; INPN: no data; additional data from Psyl'list: Algeria, France, Portugal, Slovenia, ex-Yugoslavia.

\section{COMMENTS}

Description in Rapisarda (1989). Biology in Rapisarda (1989) and Conci et al. (1996). Host-plants: several species of Rhamnus (Rhamnaceae).

\section{Trioza lautereriella Burckhardt, 1986}

Trioza lautereriella Burckhardt, 1986: 424.

Material eXAmined. - France. Alpes-Maritimes, Saorge, M10CAI2000-T7-M1, 1 은

Distribution. - Fauna Europaea: France, Germany, Italy, Slovakia, Switzerland; INPN: no data; additional data from Psyl'list: no additional data.

\section{COMMENTS}

Description in Burckhardt (1986). Biology in Conci et al. (1996). Host-plants: Angelica sylvestris L. and Daucus carota L. (Apiaceae)

\section{Trioza marginepunctata Flor, 1861}

Trioza marginepunctata Flor, 1861: 396.

Material eXamined. - France. Alpes-Maritimes, Saorge, M10CAI1400-T5-M2, 1 ㅇ (MNHN); Alpes-Maritimes, Moulinet, Col de Turini, piste de Gasta-Fume, $1625 \mathrm{~m}$, 435'ㄹ.1482”N$7^{\circ} 23^{\prime} 42.7374$ ”E, 02.VII.2008, C. Cocquempot (CBGP), 1 adult.

Distribution. - Fauna Europaea: France, Italy, Spain; INPN: no data; additional data from Psyl'list: Croatia, Hungary, Israel, Lebanon.

\section{COMMENTS}

Description and biology in Rapisarda (1989). Host-plant: Rhamnus alaternus L. (Rhamnaceae).

Trioza munda Foerster, 1848

Trioza munda Foerster, 1848: 88.

MATERIAL EXAMined. - France. Alpes-Maritimes, Bolène-Vesubie, massif de la cime du Diable, 1800-2600 m, 28.VIII.1995, $10^{7}$, I. Malenovský (MMBC).

Distribution. - Fauna Europaea: Austria, Great Britain, Bulgaria, Czech Republic, France, Italy, Moldova, Norway, Poland, Romania, Slovakia, Sweden, Switzerland, Ukraine; INPN: no data; additional data from Psyl'list: Germany, Ireland, Japan, Mongolia, Russia (Amur), Slovenia.

\section{COMMENTS}

Description in Ossiannilsson (1992). Biology in Ossiannilsson (1992) and Conci et al. (1996). Host-plants: species of the genera Knautia, Scabiosa and Succisa (Caprifoliaceae).

\section{Trioza proxima Flor, 1861}

Trioza proxima Flor, 1861: 404

Material eXAmined. - France. Alpes-Maritimes, Bolène-Vesubie, massif de la cime du Diable, 1800-2600 m, 28.VIII.1995, $10^{\text {7" }}$ I. Malenovský (MMBC); Alpes-Maritimes, La Brigue, French-Italian border between Mt Grai $(2013 \mathrm{~m})$ and Cime de Marte $(2135 \mathrm{~m})$, 21.VIII.1996, 1요 I. Malenovský (MMBC).

Distribution. - Fauna Europaea: Austria, Great Britain, Bulgaria, Czech Republic, France, Germany, Italy, Poland, Russia, Slovakia, Spain (Balearic Is.), Switzerland; INPN: no data; additional data from Psyl'list: Belgium, Slovenia.

\section{COMMENTS}

Description in Hodkinson \& White (1979) and Burckhardt (1983). Biology in Conci et al. (1996) and Lauterer \& Malenovský (2002). Host-plants: several species of Hieracium (Asteraceae)

\section{Trioza rhamni (Schrank, 1801)}

Chermes rhamni Schrank, 1801: 141.

Material eXAmined. - France. Alpes-Maritimes, Saint-Dalmasle-Selvage, M09-SES1400-T1-M2, 1 으 (MNHN).

Distribution. - Fauna Europaea: Austria, Czech Republic, Denmark, Finland, France, Germany, Great Britain, Italy, Moldova, Norway, Poland (doubtful), Romania, Russia, Slovakia, Spain, Sweden, Switzerland, Ukraine; INPN: no data; additional data from Psyl'list: Caucasus, Estonia, Hungary, Latvia, Slovenia, Turkey.

\section{COMMENTS}

Description in Hodkinson \& White (1979a) and Ossiannilsson (1992). Biology in Ossiannilsson (1992), Conci et al. 
(1996) and Lauterer (1999). Host-plants: Rhamnus cathartica L. and Rhamnus pallasii Fisch. \& C. A. Mey. (Rhamnaceae).

\section{Trioza rumicis Löw, 1880}

Trioza rumicis Löw, 1880: 557.

Material eXamined. - France. Alpes-Maritimes, Saorge, M10CAI2000-T7-M1, 1 ( (MNHN); Alpes-Maritimes, Bolène-Vesubie, massif de la cime du Diable, 1800-2600 m, 28.VIII.1995, 1 ㅇ I. Malenovský (MMBC); Alpes-Maritimes, La Brigue, French-Italian border between Mt Grai (2013 m) and Cime de Marte (2135 m), 21.VIII.1996, $50^{\pi}$, 3ㅇ, I. Malenovský (MMBC).

Distribution. - Fauna Europaea: Austria, France, Germany, Italy, Poland, Romania, Slovakia, Switzerland; INPN: no data; additional data from Psyl'list: Iran.

\section{COMMENTS}

Description in Dobreanu \& Manolache (1962) and Klimaszewski (1967). Biology in Conci et al. (1996). Host-plants: several species of Rumex (Polygonaceae).

\section{Trioza schrankii Flor, 1861}

Trioza schrankii Flor, 1861: 403.

Material eXamined. - France. Alpes-Maritimes, Saorge, M10CAI2000-T6-M1, $10^{7}$ (BMNH); same locality, M10-CAI2000-T7M1, $20^{7}$ (MNHN).

Distribution. - Fauna Europaea: Austria, Czech Republic, France, Germany, Italy, Poland, Romania, Slovakia, Switzerland, Ukraine; INPN: no data; additional data from Psyl'list: Slovenia.

\section{COMMENTS}

Description in Dobreanu \& Manolache (1962) and Klimaszewski (1967) (for T. schranki [sic]). Biology in Conci et al. (1996) and Lauterer \& Malenovský (2002). Host-plant: Astrantia major L. (Apiaceae).

Trioza senecionis (Scopoli, 1763)

Chermes senecionis Scopoli, 1763: 140.

Material eXamined. - France. Alpes-Maritimes, Saorge, M10CAI1400-T3-M1, 19 (MNHN); same locality, M10-CAI1400-T7M1, $10^{\prime \prime}, 2$ ( $170^{\star 1}, 7$ ㅇ (MNHN); same locality, M10-CAI1400-T7-M2, 27 ơ, 21 ( $(\mathrm{MNHN})$.

Distribution. - Fauna Europaea: Austria, Czech Republic, Germany, Italy, Poland, Romania, Slovakia, Switzerland, Ukraine; INPN: no data; additional data from Psyl'list: Slovenia.

\section{COMMENTS}

Description in Dobreanu \& Manolache (1962) and Klimaszewski (1967). Biology in Conci et al. (1996). Three specimens reported from France in the GBIF portal. Host-plant: Senecio fuchsii C. C. Gmel. (Asteraceae) (sometimes treated as subspecies of nemorensis) (Asteraceae). The suggestion that Adenostyles might be a host (Wagner \& Franz 1961) could not be confirmed and this should be removed from the list of host-plants of T. senecionis.

Trioza urticae (Linnaeus, 1758)

Chermes urticae Linnaeus, 1758: 454.

Material examined. - France. Alpes-Maritimes, Saint-Martin-Vésubie, M09-BOR1400-T1-M2, $10^{*}, 1$ 우 (MNHN); same locality, M09-BOR1400-T2-M2, $40^{7}$ (MNHN); same locality, M09-BOR1400-T8-M2, 1 \% (MNHN); same locality, M09-BOR1400-T1-M1, $50^{7}, 29$ (MNHN); Alpes-Maritimes, Valdeblore, M09-SES2000-T2-M2, 1 (MNHN); Alpes-Maritimes, Saorge, M10-CAI1400-T1-M1, 20", 3 \% (MNHN); same locality, M10-CAI1400-T1-M2, $10^{7}, 7$ (MNHN); same locality, M10-CAI1400-T2-M2, $10^{7}, 2$ 으 (MNHN); same locality, M10CAI1400-T3-M2, $10^{\prime \prime}$ (MNHN); same locality, M10-CAI1400-T4M2, $10^{7}$ (MNHN); same locality, M10-CAI2000-T1-M2, $10^{7}$ (MNHN); same locality, M10-CAI2000-T7-M2, 3 ㅇ (MNHN); same locality, M10-CAI2000-T2-M1, $10^{7}$ (MNHN); same locality, M10CAI2000-T2-M1, $10^{\prime \prime}$ (MNHN); same locality, M10-CAI2000-T3M1, $20^{7}$ (MNHN); same locality, M10-CAI2000-T7-M1, 50", 29 (MNHN); Alpes-Maritimes, Moulinet, Col de Turini, piste de Gasta-Fume, 1625 m, 4358'27.1482”N-7²3’42.7374"E, 02.VII.2008, C. Cocquempot (CBGP), 8 adults; Alpes-Maritimes, Moulinet, Col de Turini, $1610 \mathrm{~m}, 43^{\circ} 58^{\prime} 37.56^{\prime \prime} \mathrm{N}-7^{\circ} 23^{\prime} 30.7782^{\prime \prime} \mathrm{E}$, 09.VII.2009, on herbaceous plants, C. Cocquempot (CBGP), 13 adults; Alpes-Maritimes, Lantosque, Col d'Andrion (Col du

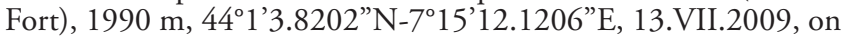
Epilobium angustifolium L., C. Cocquempot (CBGP), 10 adults; Alpes-de-Haute-Provence, Colmars-les-Alpes, Ratéry, 1670 m, $44^{\circ} 11^{\prime} 16.5804{ }^{\prime \prime} \mathrm{N}-6^{\circ} 39^{\prime} 15.4548^{\prime \prime} \mathrm{E}, 10 . \mathrm{VII} .2011$, on Urtica sp., C. Cocquempot (CBGP), 3 adults.

Distribution. - Fauna Europaea: Austria, Belgium, Bulgaria, Croatia, Cyprus, Czech Republic, Denmark, Finland, France, Germany, Great Britain, Greece, Ireland, Italy (mainland + Sardinia + Sicily), Malta, Moldova, Norway, Poland, Romania, Russia, Slovakia, Slovenia, Spain (mainland + Balearic Is.), Sweden, Switzerland, Ukraine; INPN: no data; additional data from Psyl'list: Afghanistan, Algeria, Caucasus, China, Hungary, India, Iran, Japan, Lithuania, Portugal (Madeira), Mongolia, Tadzhikistan, Turkey, West Himalaya (Uttaranchal $=$ Uttarakhand $)$

\section{COMMENTS}

Description in Hodkinson \& White (1979a) and Ossiannilsson (1992). Biology in Ossiannilsson (1992), Lauterer (1994, 2011) and Conci et al. (1996). Host-plants: several species of Urtica (Urticaceae).

Trioza velutina Foerster, 1848

Trioza velutina Foerster, 1848: 87.

Material examined. - France. Alpes-Maritimes, Saorge, M10-

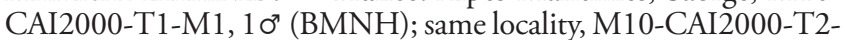
M1, $10^{7}$ (MNHN); same locality, M10-CAI2000-T3-M1, 20" (MNHN); Alpes-Maritimes, Saint-Martin-Vésubie, M09SES1400-T1-M1, 10", 2 ㅇ (MNHN). 
Distribution. - Fauna Europaea: Austria, Czech Republic, France, Germany, Great Britain, Slovakia; INPN: no data; additional data from Psyl'list: Slovenia.

\section{COMMENTS}

Description in Burckhardt \& Lauterer (2006). No precise data available on biology, since this species was confused with Trioza galii Foerster, 1848 in all works preceding the revision by Burckhardt \& Lauterer (2006). Host-plants: unknown, probably several species of Rubiaceae, as for T. galii.

\section{DISCUSSION}

The psyllids collected in 2009, 2010 and 2011 during the ATBI+M project in the Mercantour National Park (together with the four species collected by I. Malenovský in the 1990's) comprise 68 species ( $40.2 \%$ of the 169 species reported from France in Fauna Europaea). Compared to the size of the Park, which covers only c. $0.12 \%$ of Metropolitan France, these results reflect the importance of such protected area for preserving ecosystems, especially in groups like psyllids that are closely dependant on the presence and abundance of their host-plants.

Seven of the species are new for France. In the case of Craspedolepta artemisiae, the data from Mercantour represent the westernmost record. Craspedolepta nebulosa, Cacopsylla propinqua and Cyamophila prohaskai were already known from the Swiss (Burckhardt 1994b; Burckhardt et al. 2007; Schaefer 1949) and Italian Alps (Conci et al. 1993). Trioza flixiana is also known from the Swiss Alps (Burckhardt \& Lauterer 2002b), but shows a larger area of distribution (Pyrenees, Spanish Basque Country; GBIF data) at a wider range of altitudes (Spain and Czech Republic). Bactericera parastriola was also known from the Swiss and Italian Alps, but also from Poland, Romania and Sweden (Conci et al. 1988), from Austria (Burckhardt \& Lauterer 1997) and, more recently, from the Czech Republic (Lauterer \& Malenovský 2002), indicating a boreomontane distribution. The presence of Eryngiofaga refuga, otherwise known only from Kazakhstan and West Siberia, remains to be confirmed, since only one male has been collected from Mercantour.

The range of altitudes in the Park, from 490-3143 m a.s.l., makes the potential psyllid fauna even richer than 67 species in the area, since only altitudes from 1421-2058 m have been sampled. That is why we find here several orophilous species like Psylla alpina (Pyrenees, Alps, Carpathians), Trioza caesaris (Alps), Trioza schrankii (Massif Central, Alps, Carpathians), Trioza brachyceraea (Massif Central, Alps), Trioza senecionis (Alps, Carpathians) and Aphalara sauteri (Alps and Andalusia according to Burckhardt \& Lauterer 1997).

Other orophilous species found in the park that show a larger area of distribution, including Fennoscandia and/or expanding eastward, are: Bactericera femoralis, Bactericera bohemica, Cacopsylla rhododendri, Psylla fusca, Trioza abdominalis, Trioza cerastii and Trioza proxima. Livilla pyrenaea is present in the Pyrenees and the Alps, as well as in Algeria.
Two species, Ctenarytaina eucalypti and Acizzia uncatoides, originate from Australia and have been introduced into France on Eucalyptus (in the early 1980s) and on Acacia and Albizzia, respectively. Despite the presence of two males in a Malaise trap in this study, most populations of C. eucalypti have been controlled in eastern-southern France by the introduction of a specific parasitoid wasp, Psyllaephagus pilosus Noyes, 1988 (Malausa \& Girardet 1997; Hodkinson 1999). Calophya rhois originates from East Asia, as does its host (Cotinus coggygria), and has probably been naturalised in France for a long time. Livilla variegata probably originates from eastern Europe, and its wide current distribution may be due to the use of Laburnum as an ornamental tree in many places in Europe. Another eucalyptus-feeding species originating from Australia, Glycaspis brimblecombei Moore, 1964, has recently been discovered in France (Cocquempot et al. 2012), in the southern part of the Alpes-Maritimes department, and could also be present in the Mercantour park on Eucalyptus camaldulensis Dehnh.

Several other orophilous species known from France have not been collected in the Mercantour, which may be due to the collecting methods used (mainly Malaise traps and limited net sweeping): Cacopsylla corcontum (Šulc, 1909), C. elegantula (Zetterstedt, 1840), C. hippophaes (Foerster, 1848), C. myrtilli (Wagner, 1947), Bactericera bucegica (Dobreanu \& Manolache, 1962), Trioza chrysanthemi Löw, 1878, T. dispar Löw, 1878, T. laserpitii Burckhardt \& Lauterer, 1982, T. saxifragae Löw, 1888, T. rotundata Flor, 1861 and T. tatrensis Klimaszewski, 1965.

Future fieldwork in the Mercantour National Park may lead to the discovery of several alpine or subalpine species never collected in France before, but present in bordering countries (Switzerland and Italy), such as Livilla vicina (Löw, 1886), L. vittipennella (Reuter, 1875), Bactericera harrisoni (Wagner, 1955), Trioza remaudierei Burckhardt \& Lauterer, 2002, the rare T. megacerca Burckhardt, 1983 and T. tripteridis Burckhardt, Conci, Lauterer \& Tamanini, 1991.

Cacopsylla iteophila is a rare and localised species (Conci \& Tamanini 1990b) that feeds on Salix.

The most abundant species in Malaise traps, which are rarely used for collecting Psylloidea, were Livilla pyrenaea, Trioza senecionis and Cacopsylla brunneipennis. In sweep-net samples, the most common species were Cacopsylla sorbi and C. artemisiae (>100 specimens), followed by Psyllopsis fraxinicola, Trioza centranthi and C. brunneipennis.

\section{Acknowledgements}

The authors are grateful to Isabelle Meusnier and Antonin David (both CBGP, Montpellier, France) for processing molecular analyses (unpublished), and to Pavel Lauterer (Moravian Museum, Brno, Czech Republic) for confirming identifications. Special thanks to Igor Malenovský (Moravian Museum and Masaryk University, Brno, Czech Republic) for helpful review of this manuscript and for allowing us to add his own specimen data from the Mercantour. 


\section{REFERENCES}

Bain C., Labit B., Mimaud J. \& Tanguy M. 1976. — Résultats de l'expérimentation effectuée en 1975 par le service de la protection des végétaux. II. Lutte contre les ravageurs et les maladies. Phytoma 28 (282): 7-13.

BALACHOWSKY A. \& MESNIL L. 1935. — Les insectes nuisibles aux plantes cultivées. t. 1. Etablissements Busson, Paris: 1136 p.

BÉGUINOT J. 2002. - Contribution au recensement de la faune cécidogène d'altitude dans le bassin de l'Ubaye (Alpes sud-occidentales françaises). Bulletin de la Société linnéenne de Lyon, 71 (2): 65-74.

BIN F. 1972. - Presenza in Italia della Trioza femoralis Foerster (Rhynchota, Psyllidae) e notizie su due Chalcidoidei suoi parassiti. Entomologica 8: 45-53.

BuRCKHARDT D. 1983. - Beiträge zur Systematik und Faunistik der schweizerischen Psyllodea (Sternorrhyncha). Entomologica Basiliensia 8: 43-83.

BURCKHARDT D. 1986. - Taxonomy and host plant relationships of the Trioza apicalis Forster complex (Hemiptera, Homoptera: Triozidae). Entomologica scandinavica 16 (4): 415-432.

BurCKHARDT D. 1994a. - Psylloid pests of temperate and subtropical crop and ornamental plants (Hemiptera, Psylloidea): A review. Entomology (Trends in Agricultural Science) 2: 173-186.

BurCKHARDT D. 1994b. - Notes sur la répartition en Suisse de quelques espèces de psylles (Hemiptera, Psylloidea). Bulletin Romand d'Entomologie 12 (2): 109-115.

BuRCKHARDT D. 1998. - Ctenarytaina eucalypti (Maskell) (Hemiptera, Psylloidea) neu für Mitteleuropa mit Bemerkungen zur Blattflohfauna von Eucalyptus. Mitteilungen der entomologischen Gesellschaft Basel 48 (2): 59-67.

BurCKHARDT D. 1999. - Cacopsylla pulchella (Löw), eine Blattflohart des Judasbaum, auch in Basel (Hemiptera, Psylloidea). Mitteilungen der entomologischen Gesellschaft Basel 49 (2): 71-76.

BurCKHARDT D. 2004. - Fauna Europaea: Psylloidea. Fauna Europaea version 1.0, http://www.faunaeur.org

BurCKHARDT D. 2010. - Pictorial key of Central European Cacopsylla species associated with Rosaceae, http://www.psyllidkey.com/index.html

BurCKHARDT D. \& HodKInSON P. 1986. - A revision of the west Palaearctic pear psyllids (Hemiptera: Psyllidae). Bulletin of Entomological Research 76 (1): 119-132.

BurCKHARDT D. \& LAUTERER P. 1997. - A taxonomic reassessment of the triozid genus Bactericera (Hemiptera: Psylloidea). Journal of Natural History 31(1): 99-153.

BURCKHARDT D. \& LAUTERER P. 2002a. - Revision of the central European Trioza rotundata Flor complex (Hemiptera, Psylloidea): taxonomy and bionomy. Mitteilungen der schweizerischen entomologischen Gesellschaft 75 (1-2): 21-34.

BurckHARDT D. \& LAUTERER P. 2002b. - Trioza fixiana sp. n. (Hemiptera, Psylloidea), a new jumping plant-louse species from Central Europe. Revue suisse de Zoologie 109 (4): 799-802.

Burckhardt D. \& Lauterer P. 2006. - The Palaearctic triozids associated with Rubiaceae (Hemiptera, Psylloidea): a taxonomic re-evaluation of the Trioza galii Foerster complex. Revue suisse de Zoologie 113 (2): 269-286.

BURCKHARDT D. \& OUVRARD D. 2012. - A revised classification of the jumping plant-lice (Hemiptera: Psylloidea). Zootaxa 3509: 1-34.

BurCKHARDT D., MÜHLETHALER R., LAUTERER P., MaLeNOVSKÝ I. \& KunZ G. 2007. — Die Blattfl̈he und Zikaden der Alp Flix (Insecta, Hemiptera: Psylloidea und Auchenorrhyncha). Jahresbericht der Naturforschenden Gesellschaft Graubünden 114: 91-97.

Carraro L., Osler R., Loi N., Ermacora P. \& Refatti E. 1998. - Transmission of european stone fruit yellows phytoplasma by Cacopsylla pruni. Journal of Plant Pathology 80: 233-239.

Cocquempot C., Malausa J.-C., Thaon M. \& Brancaccio L. 2012. — Le Psylle du Gommier rouge (Glycaspis brimblecombei Moore) introduit sur les eucalyptus de France (Hemiptera, Psyllidae). Bulletin de la Société entomologique de France 117 (3): 363-370.
Conci C. \& TAmanini L. 1982. - Psylla limbata, nuova per l'Italia, da Rhamnus alpinus (Homoptera Psylloidea). Atti della Societa Italiana di Scienze Naturali e del Museo Civico di Storia Naturale in Milano 123 (4): 483-494.

Conci C. \& TAMANini L. 1984. - Trioza (Trioza) rapisardai n. sp., from Piemonte, host plant Laserpitium siler (Homoptera Psylloidea). Atti della Societa Italiana di Scienze Naturali e del Museo Civico di Storia Naturale in Milano 125 (3-4): 201-208.

CONCI C. \& TAMANINI L. 1985. - Bactericera harrisoni in Italy, and comparison with B. bohemica (Homoptera Psylloidea). Annali dei Musei civici, Rovereto 1: 99-110.

CONCI C. \& TAMANini L. 1986a. - Cacopsylla propinqua, from Valle d'Aosta, new for Italy (Homoptera Psylloidea). Doriana 6: 1-8.

Conci C. \& TAMANini L. 1986b. - Cyamophila prohaskai from Alto Adige and Trentino, genus and species new for Italy (Homoptera: Psylloidea). Studi Trentini di Scienze Naturali Acta Biologica 62: 59-68.

Conci C. \& TAMAnini L. 1988. — Seven species of psylloidea new for Italy. Annali dei Musei civici, Rovereto 4: 307-320.

CONCI C. \& TAMANINI L. 1989a. - Life history, nymphs and egg of Cyamophila prohaskai, host plant Anthyllis vulneraria ssp. alpestris. Studi Trentini di Scienze Naturali, Acta Biologica 65 (1988): 137-146.

CONCI C. \& TAMANINI L. 1989b. - Rare or interesting species of Italian Psylloidea. I. Atti dell'Academia Roveretana degli Agiati 238 (1988): 47-72.

Conci C. \& TAMAnini L. 1990a. - Notes on the genus Psyllopsis (Homoptera Psylloidea). Atti della Accademia Roveretana degli Agiati 29: 57-85.

Conci C. \& TAmanini L. 1990b. - Cacopsylla iteophila in Alto Adige and Trentino, new for Italy (Homoptera Psylloidea). Annali dei Musei civici, Rovereto 5 (1989): 205-218.

CONCI C., OssianniLsson F. \& TAMANINI L. 1988 - Bactericera parastriola sp. n., from Salix phylicifolia, lapponum and waldsteiniana, in Sweden and Italy (Homoptera Psylloidea). Atti della Società italiana di scienze naturali e del museo civico di storia naturale di Milano 129 (2-3): 225-236.

CONCI C., RAPISARDA C. \& TAMANINI L. 1993. - Annotated catalogue of the Italian Psylloidea. First part. (Insecta Homoptera). Atti della Accademia Roveretana degli Agiati Serie 7 B Classe di Scienze Matematiche Fisiche e Naturali 2B: 33-135.

CONCI C., RAPISARDA C. \& TAMANINI L. 1996. - Annotated catalogue of the Italian Psylloidea. Second part (Insecta Homoptera). Atti della Accademia Roveretana degli Agiati Serie 7 B Classe di Scienze Matematiche Fisiche e Naturali 5B: 5-207.

Cотте J. 1915. - Une randonnée dans les Basses-Alpes. Annales du Muséum d'Histoire naturelle de Marseille, 15 (3): 21 p.

Deharveng L., Bedos A., Daugeron C., Villemant C. \& JudSON M. L. I. 2015. — Organization, usefulness and limitations of an ATBI (All Taxa Biodiversity Inventory): the inventory of terrestrial invertebrates in the Mercantour National Park, in Daugeron C., Deharveng L., Isaia M., Villemant C. \& Judson M. (eds), Mercantour/Alpi Marittime All Taxa Biodiversity Inventory. Zoosystema 37 (1): 9-30. http://dx.doi.org/10.5252/ z2015n1a1

Dobreanu E. \& MANOLAChe C. 1959. — Nouvelle contribution à la connaissance des Psylles (Psylloidea-Triozinae). Revue de Biologie 4: 279-299.

Dobreanu E. \& Manolache C. 1962. - Insecta. Homoptera Psylloidea. Fauna Republicii Populare Romîne. Vol. 8, Fascicula 3. Academiei Republicii Populare Romîne, Bucuresti, 377 p.

HodKINSON I. D. 1999. - Biocontrol of eucalyptus psyllid Ctenarytaina eucalypti by the Australian parasitoid Psyllaephagus pilosus: a review of current programmes and their success. Biocontrol News and Information 20 (4): $129 \mathrm{~N} 134 \mathrm{~N}$.

HodKINSON I. D. \& WhITE I. M. 1979a. - Homoptera Psylloidea. Handbooks for the Identification of British Insects, Volume 2, Part 5(a). Royal Entomological Society of London, London, 98 p. Hodkinson I. D. \& White I. M. 1979b. — New psyllids from 
France with redescriptions of the type species of Floria Löw and Amblyrhina Löw (Homoptera: Psylloidea). Entomologica scandinavica 10 (1): 55-63.

HodKINSON I. D. \& Hollis D. 1980. - Floria variegata Löw (Homoptera: Psylloidea) in Britain. Entomologists Gazette 31 (3): 171, 172.

HodKInson I. D. \& Hollis D. 1981. — The psyllids (Homoptera: Psylloidea) of Mallorca. Entomologica scandinavica 12 (1): 65-77.

HodKInSON I. D. \& Hollis D. 1987. — The legume-feeding psyllids (Homoptera) of the west Palaearctic region. Bulletin of the British Museum (Natural History) Entomology series 56 (1): 1-86.

KlimasZeWSKI S. M. 1963. - Polnische Arten der Gattung Psylla Geoff. (Homoptera, Psyllidae). Annales Zoologici (Warsaw) 20 (20): 363-455.

KLIMASZEWSKI S. M. 1967. — Polnische Arten der Familie Triozidae (Homoptera: Psyllodea). Annales Zoologici (Warsaw) 25 (3): 227-315.

KLIMASZEWSKI S. M. 1983. - Revision of the Palaearctic species of the genus Craspedolepta Enderl. s. 1. (Homoptera, Aphalaridae). Polskie Pismo Entomologiczne 53 (1-2): 3-29.

Konovalova Z. A. 1988. - 2. Suborder Psyllinea, in LeR P. A. (ed.), Key to the Identification of Insects of the Soviet Far East, Volume 2, Homoptera and Heteroptera. Nauka, Leningrad: 495-540.

LAUTERER P. 1976. — Psyllids of Wetland Nature Reserves of the German Democratic Republic, with Notes on Their Biology, Taxonomy and Zoogeography (Homoptera, Psylloidea). Faunistische Abhandlungen (Staatliches Museum für Tierkunde in Dresden) 6 (10): 111-122.

LAUTERER P. 1979. - New and interesting records of psyllids from Czechoslovakia (Homoptera, Psylloidea). Acta Musei Moraviae Scientiae Naturales 64: 93-102.

LAUTERER P. 1993. - Notes on the bionomics and occurrence of some psyllids (Homoptera, Psylloidea) in Czechoslovakia and the Balkan Peninsula. Acta Musei Moraviae Scientiae Naturales 77 (1-2): 147-156.

Lauterer P. 1994. - Psyllids (Homoptera, Psylloidea) from the area flooded by the Nove Mlyny reservoir system and its environs in southern Moravia. Acta Musei Moraviae Scientiae Naturales 78 (1-2): 165-200.

LAUTERER P. 1998. - Results of the investigations on Hemiptera in Moravia, made by the Moravian museum (Introduction, Psylloidea I). Acta Musei Moraviae Scientiae Biologicae 83 (1-2): 99-126.

LAUTERER P. 1999. - Results of the investigations on Hemiptera in Moravia, made by the Moravian museum (Psylloidea 2). Acta Musei Moraviae Scientiae Biologicae 84 (1-2): 71-151.

Lauterer P. 2011. - Psyllids (Hemiptera, Psylloidea) inhabiting the canopy and shrub layer of an oak and hornbeam forest in Báb near Nitra (Slovakia). Folia Faunistica Slovaca 16 (2): 97-101.

LAUTERER P. \& MALENOVSKÝ I. 2002. - New distributional and biological data on European Psylloidea (Hemiptera, Sternorrhyncha), with special reference to the fauna of the Czech Republic and Slovakia. Entomologica Basiliensia 24: 161-177.

LoGinova M. M. 1963. - Revision of the species of the genera Aphalara Frst. and Craspedolepta Enderl. (Homoptera, Psylloidea) in the fauna of the USSR. II. Entomological Review 42 (3):334-346.

Loginova M. M. 1966. - New and little-known Psyllids (Homoptera, Psylloidea) from the USSR. Trudy Zoologicheskogo Instituta 37: 3-35.

Loginova M. M. 1975. - Representatives of the genus Psylla (Homoptera, Psylloidea) developing on Rhamnus and Cerasus. Zoologichesky Zhurnal 54 (5): 701-711.

Malausa J. C. \& GiRARDET N. 1997. — Lutte biologique contre le psylle de l'eucalyptus: acclimatation sur la Côte d'Azur d'un auxiliaire prometteur, Psyllaephagus pilosus. Phytoma - La Défense des Végétaux 498: 49-51.
MALENOVSKÝ I. \& KMENT P. 2004. - First record of Livilla variegata (Low, 1881) (Hemiptera: Psylloidea, Psyllidae) in Slovakia. Biologia (Bratislava) 59 (2): 292.

Mercier L. \& Poisson R. 1926. - Un Hémiptère Homoptère, de la famille des Psyllines, parasite d'Eucalyptus cultivés à Cherbourg. Bulletin de la Société Linnéenne de Normandie Series 7 (9): 34-37.

Ossiannilsson F. 1992. - The Psylloidea (Homoptera) of Fennoscandia and Denmark. Fauna Entomologica Scandinavica, Volume 26. E. J. Brill, Leiden, New York, 346 p.

Ouvrard D. 2014. - Psyl'list - The World Psylloidea Database. http://www.hemiptera-databases.com/psyllist - searched on 24 March 2014

Peccoud J., Labonne G. \& Sauvion N. 2013. - Molecular Test to Assign Individuals within the Cacopsylla pruni Complex. PLoS ONE 8 (8): e72454.

Peragallo A. 1881. - Insectes nuisibles aux oliviers. Annales de la Société entomologique de France, Bulletin des Séances, 1881: LXXI-LXXII.

Queiroz D. L., Burckhardt D. \& Majer J. 2012. — Integrated Pest Management of Eucalypt Psyllids (Insecta, Hemiptera, Psylloidea), in LARRAMENDY M. L. \& SOLONESKI S. (eds), Integrated Pest Management and Pest Control - Current and Future Tactics. InTech, Rijeka: 385-412.

RAPISARDA C. 1987. - Nymphal description and biological notes on some species of psyllids new for the Italian fauna (Homoptera, Psylloidea). Phytophaga 2 (1984): 57-72.

RAPISARDA C. 1989. - Contributo alla conoscenza degli Psilloidei viventi su Rhamnus alaternus L. in Italia (Homoptera). Phytophaga 3 (1985-89): 21-60.

RAPISARDA C. \& CONCI C. 1989. - Faunistic notes and zoogeographical considerations on the psyllid fauna of the south-eastern Alps. Biogeographia 13: 623-639.

Risso A. 1826. - Histoire naturelle des principales productions de l'Europe méridionale et particulièrement de celles des environs de Nice et des Alpes-Maritimes, 5. Librairie Levrault, Paris \& Strasbourg: $400 \mathrm{p}$.

SCHAEFER H. A. 1949. - Beiträge zur Kenntnis der Psylliden der Schweiz. Mitteilungen der schweizerischen entomologischen Gesellschaft 22 (1): 1-96.

Seljak G., MalenovskÝ I. \& Lauterer P. 2008. — New records of jumping plant-lice from Slovenia with description of Bactericera lyrata sp. n. (Hemiptera: Psylloidea). Revue suisse de Zoologie 115 (3): 527-540.

ŠUlC K. 1913. - Monographia generis Trioza Foerster. Species regionis palaearcticae. Pars IV., No. 36.-49. Sitzungsberichte der königlichen böhmischen Gesellschaft der Wissenschaften 1913 (1): $1-48$.

TAMANINI L. 1977. - Notizie corologiche e morfologiche su alcuni psillidi poco noti delle prealpi (Homoptera Psyllodea). Studi Trentini di Scienze Naturali Acta Biologica 54: 103-119.

VONDRÁČEK K. 1950. - To the revision of Melichar's collection of jumping plant lice (Psyllinea). Acta Musei Moraviae 35: 183-189.

VONDRÁČEK K. 1957. - Mery-Psylloidea (Fauna CSR, Svazek 9). [Jumping plant lice - Psylloidea. (Fauna of Czechoslovakia, Volume 9)]. Ceskoslovenska Akademie Ved., Praha, 431 p.

WAGNER W. 1947. - Neue deutsche Homopteren und Bemerkungen über schon bekannte Arten. Verhandlungen des Vereins für naturwissenschaftliche Heimatforschung zu Hamburg 29 (1947): 72-89.

WAGNER W. \& Franz H. 1961. - Überfamilie Sternorrhyncha (Psylloidea), in Franz, H. (ed), Die Nordost-Alpen im Spiegel Ihrer Landtierwelt. Eine Gebietsmonographie umfassend: Fauna, Faunengeschichte, Lebensgemeinschaften und Beeinflussung der Tierwelt durch den Menschen, Vol. 2. Universitätsverlag Wagner, Innsbruck: 158-179. 